\title{
NeuroRegulation
}

\section{Proceedings of the 2014 ISNR Conference}

\author{
Selected Abstracts of Conference Presentations at the 2014 International Society \\ for Neurofeedback and Research (ISNR) $22^{\text {nd }}$ Conference, Bayside San Diego, \\ California, USA
}

Copyright: (c) 2014. ISNR. This is an Open Access article distributed under the terms of the Creative Commons Attribution License (CC-BY).

\section{KEYNOTE PRESENTATIONS}

\author{
Harnessing Brain Plasticity: The Future of Neurotherapeutics \\ Adam Gazzaley, MD, PhD \\ Neuroscience Imaging Center and Neuroscape Lab \\ Principal Investigator, Gazzaley Lab, University of California, San Francisco, California, USA
}

\begin{abstract}
A fundamental challenge of modern society is the development of effective approaches to enhance brain function and cognition in both healthy and impaired individuals. For the healthy, this serves as a core mission of our educational system and for the cognitively impaired this is a critical goal of our medical system. Unfortunately, there are serious and growing concerns about the ability of either system to meet this challenge. I will describe an approach developed in our lab that uses custom-designed video games to achieve meaningful and sustainable cognitive enhancement (e.g., Anguera et al., 2013), as well the next stage of our research program, which uses video games integrated with technological innovations in software (e.g., brain computer interface algorithms, Neurofeedback, GPU computing) and hardware (e.g., virtual reality headsets, mobile EEG, transcranial electrical brain stimulation) to create a novel personalized closed-loop system. I will share with you a vision of the future in which video games serve as an underlying engine to enhance our brain's information processing systems, thus reducing our reliance on non-specific drugs to treat neurological and psychiatric conditions and allowing us to better target our educational efforts.
\end{abstract}

\section{Online, Voluntary Control of Individual Neurons in the Human Brain \\ Moran Cerf, PhD \\ Assistant Professor of Business and Neuroscience, Kellogg School of Management, Illinois, USA and the University of California, Los Angeles, Department of Neurosurgery, California, USA}

Recording from single neurons in patients implanted with intracranial electrodes for clinical reasons, I will demonstrate that humans can regulate the activity of their neurons in the medial temporal lobe (MTL) to alter the outcome of the contest between external images and their internal representation using feedback from these neurons. I will discuss some of the works in real-time recording of the activity of individual neurons in the brains of humans in the last decade, and how these can be used to access the underlying mechanisms of our decisions, emotion, memory, and free will. 


\title{
KEYNOTE PANEL
}

\section{Experiences and Opportunities of Brain Training for Athletes}

\author{
Moderator: Leslie Sherlin, PhD, SenseLabs \\ Panel: Carlos Quentin, Outfielder, San Diego Padres \\ Kaya Turski, Canadian Freestyle Skier, X Games \\ Dan Buchner, Professional Golfer
}

\begin{abstract}
Athletic performance has in the past focused on training the body. However, an athlete who can exercise volitional control of the levels of cognitive engagement and arousal of brain and body has an advantage during competition. A growing interest in a quantified self and the technological advances contributing to wearable sensors are creating a new opportunity in the field of sport psychology. The panel moderator will provide a brief overview of the most recent trends in the implementation of electroencephalography evaluation and training methods in professional and world-class level athletics. The panel will host professional athletes of wide-ranging demographics who have utilized these techniques to help them achieve the highest levels of success in the world of sports. Case-specific data of psychophysiological and performance outcomes will be elaborated upon as each athlete will describe his or her experiences of the evaluation and training. Through interview style discussion, they will discuss the reasons they chose to implement these techniques and the benefits they received from the training.
\end{abstract}

\section{INVITED PRESENTATIONS}

\section{An Introduction to Misophonia: Case Reports and Physiological Findings}

Miren Edelstein, Doctoral Student

University of California, San Diego, California, USA

Misophonia, literally translated to "hatred of sound," is a little known condition in which certain sounds trigger an involuntary "fight or flight" response in certain individuals. These sounds are often repetitive, innocuous, and social in nature (gum chewing, pen clicking, lip smacking). When exposed to these trigger sounds, misophonic individuals report intense feelings of anger, panic, and rage to the point where their everyday lives can be severely impacted. Here, I describe case reports and provide preliminary physiological evidence (via skin conductance) suggesting that misophonia evokes heightened autonomic responses to certain sounds not seen in control subjects.

\section{Altered Cerebral Connectivity and the Corpus Callosum: Adaptation or Dysfunction \\ Elliott Sherr, MD, PhD \\ Professor of Neurology and Pediatrics, University of California, San Francisco, California, USA}

Agenesis of the corpus callosum (ACC) is a common disruption in brain development, which is often associated with cognitive and behavioral deficits including autism, intellectual disability, and disruptions in emotional regulation. There is also considerable variation in the expression of these phenotypes in different cases of ACC, suggesting that understanding the 
pathways implicated and the genetics that underlie the causes of ACC may shed light on how altered connectivity leads to both pathology and adaptive responses.

\section{Double-Blind Randomized Clinical Trial of Neurofeedback for ADHD: Rationale and Strategy}

Roger deBeus, PhD

University of North Carolina, Asheville, North Carolina, USA

Objective. Current established, evidence-based treatments for attention-deficit/hyperactivity disorder (ADHD) are incompletely effective and not universally acceptable, and appear to wane in effect over time despite significant immediate benefit. Additional treatments are needed that are effective with persisting benefit, preferably related to a biomarker predicting treatment response. A good candidate is neurofeedback (NF) based on observations that patients with ADHD often have excessive theta band $(4-8 \mathrm{~Hz})$ quantitative EEG power, low beta band (13-21 Hz) power, and excessive theta-beta ratio (TBR). Although there has been an explosion of ADHD NF research over the past decade, overall efficacy remains inconclusive.

Method. Experts in NF, ADHD, clinical trials, statistics, and data management have joined to design a double-blind sham-controlled randomized clinical trial. This 5-year study has been funded by the National Institutes of Mental Health (NIMH). At each of two sites (one university and one NF clinic) 70 children (total $N=140$ ) ages 7 through 10 with rigorously diagnosed moderate to severe ADHD and TBR $>5$ will be randomized in a $3: 2$ ratio to active TBR down-training by NF versus a sham training of equal duration, intensity, and appearance. Multi-domain assessments at baseline, mid-treatment, treatment end, and follow-ups at 6 months, 1 year, and 2 years will include parent and teacher ratings of symptoms and impairment, neuropsychological tests, clinician ratings, and quantitative EEG, as well as tests of blinding and of sham inertness.

Results and Conclusions. Hypotheses include that NF will improve parent- and teacherrated inattentive symptoms (primary outcome) and other outcomes more than sham, that benefit will persist for 2 years after training, that initial TBR will moderate treatment response, and that change in TBR will mediate response. Research Domain Criteria and EEG brain changes will be explored, including relationship of TBR to clinical symptoms, executivefunction impairment, and sleep.

\section{STUDENT TRAVEL AWARD WINNERS}

\section{Prefrontal Neurofeedback Training Approaches in Autism}

Yao Wang, MS, Estate Sokhadze, PhD, Ayman El-Baz, PhD, Lonnie Sears, PhD, Allan Tasman, MD, and Manuel Casanova, MD

University of Louisville, Kentucky, USA

Background. Neurofeedback (NFB) has a potential as an intervention for autism spectrum disorder (ASD) treatment. Neurofeedback applications for attention-deficit/hyperactivity disorder (ADHD) has recently been reviewed extensively (Arns, de Ridder, Strehl, Breteler, \& 
Coenen, 2009; Gevensleben et al., 2009; Sherlin, Arns, Lubar, \& Sokhadze, 2010). Several publications suggest that ADHD protocols may also be efficacious for the treatment of autism (Kouijzer, de Moor, Gerrits, Buitelaar, \& van Schie, 2009; Kouijzer, van Schie, de Moor, Gerrits, \& Buitelaar, 2010). However, strategies commonly used in ADHD treatment (suppression of the frontal theta, enhancement of the SMR or slow beta) cannot be duly transferred to ASD treatment. Coben's studies (Coben \& Padolsky, 2007; Coben \& Myers, 2010; Coben, Linden, \& Myers, 2010) along with Scolnick (2005) and Jarusiewicz (2002) findings point at the preference of individualized protocols that use qEEG-guided intervention targets.

Objectives. We believe that approach in autism neurotherapy should focus on prefrontal NFB training, which may result in better executive functions. We selected two protocols: one targeting suppression of high amplitude (Inhibit All protocol [IA], i.e., wideband suppression) across all EEG bands (typically used in ADHD), while the second one targeting increase of the relative power of $40 \mathrm{~Hz}$ centered gamma (gamma [RG]) in addition to Inhibit All at $\mathrm{FPz}$ site. The current pilot study is aimed to compare effectiveness of two prefrontal NFB protocols in autism. Development of more refined prefrontal NFB protocol for ASD was one of the main goals.

Method. The study participants with ASD diagnosis were recruited from Department of Pediatrics. In first group $(N=8, M=14.9$ years) we used twelve 25-minute long sessions of weekly IA protocol, while in the second group $(N=15, M=13.6$ years) we used gamma upregulation $[R G$ ] protocol (25 min, 12 sessions) using PAT device. Feedback was arranged in a form of auditory feedback and visual feedback (bars, lines) with information about values of controlled parameters, their relation to the threshold (e.g., \% over threshold), and other descriptors of the training progress. We also used control of DVD by training parameters (e.g., controlling size and brightness of video by EEG feedback). Analysis of EEG during NFB training was conducted for relative power of bands and several ratios during 25 minutes of each session and across the course of neurotherapy as it was reported in our study on ADHD (Hillard, El-Baz, Sears, Tasman, \& Sokhadze, 2013).

Results. The trainees in IA protocol were successful in reducing theta/beta and theta/alpha ratios, but effects were not transferred from session to session. The second protocol-aimed to target increase of relative gamma power-resulted both in reduction of slow wave proportion and increase of higher frequency rhythms power (high beta, gamma) and decrease of theta/beta and theta/gamma ratios, not only during the session but also across sessions. This group also showed EEG changes that were typical for the IA group (i.e., decreased theta/slow beta ratio).

Conclusion. Application of prefrontal neurofeedback protocols aimed to increase higher frequency activity (beta, gamma) along with suppression of slow frequencies (theta) could be considered as a promising approach that warrants further investigation using as outcome measures not only EEG changes during NFB, but also clinical behavioral measures and quantitative EEG outcomes. 


\section{References}

Arns, M., de Ridder, S., Strehl, U., Breteler, M., \& Coenen, A. (2009). Efficacy of neurofeedback treatment in ADHD: The effects of inattention, impulsivity and hyperactivity: A meta-analysis. Clinical EEG and Neuroscience, 40(3), 180-189. http://dx.doi.org/10.1177/155005940904000311

Coben, R., \& Padolsky, I. (2007). Assessment-guided neurofeedback for autistic spectrum disorder. Journal of Neurotherapy, 11(1), 5-23. http://dx.doi.org/10.1300/J184v11n01_02

Coben, R., \& Myers, T. E. (2010). The relative efficacy of connectivity guided and symptom based EEG biofeedback for autistic disorders. Applied Psychophysiology and Biofeedback, 35(1), 13-23. http://dx.doi.org/10.1007/s10484-009-9102-5

Coben, R., Linden, M., \& Myers, T. E. (2010) Neurofeedback for autistic spectrum disorder: A review of the literature. Applied Psychophysiology and Biofeedback, 35(1), 83-105. http://dx.doi.org/10.1007/s10484-009-9117-y

Gevensleben, H., Holl, B., Albrecht, B., Vogel, C., Schlamp, D., Kratz, O., ... Heinrich, H. (2009). Is neurofeedback an efficacious treatment for ADHD? A randomised controlled clinical trial. The Journal of Child Psychology and Psychiatry, 50(7), 780789. http://dx.doi.org/10.1111/j.1469-7610.2008.02033.x

Hillard, B., El-Baz, A. S., Sears, L., Tasman, A., \& Sokhadze, E. M. (2013). Neurofeedback training aimed to improve focused attention and alertness in children with ADHD: A study of relative power of EEG rhythms using custom-made software application. Clinical EEG and Neuroscience, 44(3), 193-202. http://dx.doi.org/10.1177/1550059412458262

Jarusiewicz, B. (2002). Efficacy of neurofeedback for children in the autistic spectrum: A pilot study. Journal of Neurotherapy, 6(4), 39-49.

http://dx.doi.org/10.1300/J184v06n04_05

Kouijzer, M. E. J., de Moor, J. M. H., Gerrits, B. J. L., Buitelaar, J. K., \& van Schie, H. T. (2009). Long-term effects of neurofeedback treatment in autism. Research in Autism Spectrum Disorders, 3(2), 496-501. http://dx.doi.org/10.1016/j.rasd.2008.10.003

Kouijzer, M. E. J., van Schie, H. T., de Moor, J. M. H., Gerrits, B. J. L., \& Buitelaar, J. K. (2010). Neurofeedback treatment in autism. Preliminary findings in behavioral, cognitive, and neurophysiological functioning. Research in Autism Spectrum Disorders, 4(3), 386-399. http://dx.doi.org/10.1016/j.rasd.2009.10.007

Scolnick, B. (2005). Effects of electroencephalographic biofeedback with Asperger's syndrome. International Journal of Rehabilitation Research, 28(2), 159-163. http://dx.doi.org/10.1097/00004356-200506000-00010

Sherlin, L., Arns, M., Lubar, J., \& Sokhadze, E. (2010). A position paper on neurofeedback for the treatment of ADHD. Journal of Neurotherapy, 14(2), 66-78. http://dx.doi.org/10.1080/10874201003773880 


\title{
Transcranial Direct Current Stimulation Improves Visual and Spatial Aspects of Memory in Major Depression
}

\author{
Mohammad Ali Salehinejad, $M A^{1}$, Reza Rostami, $M D^{2}$, and Elham Ghanavati, $M A^{3}$ \\ ${ }^{1}$ University of Kansas, Kansas, USA \\ ${ }^{2}$ University of Tehran, Iran \\ ${ }^{3}$ Islamic Azad University, Iran
}

With a lifetime prevalence estimated at $16 \%$, major depression (MD) is a major public health issue. Previous studies have shown that depression has been associated with a variety of cognitive impairments. In addition to cognitive impairments, MD is usually accompanied by alterations of cortical activity, especially in prefrontal areas. Recent studies have highlighted the importance of noninvasive brain stimulation as a means of modulating cortical excitability. Recent studies on MD have revealed that transcranial direct current stimulation (tDCS) induces cortical excitability, which facilitates memory and especially working memory. On the other hand, visual aspects of memory in MD have not been yet investigated.

Objective. This study aimed to investigate whether anodal and cathodal tDCS applied over dorsolateral prefrontal cortex (DLPFC), would significantly improve visual memory in patients with MD.

Methods. Thirty $(N=30)$ patients with MD were randomly assigned to receive either experimental (active) or control (sham) tDCS. The participants underwent a series of visual memory tasks before and after 10 sessions of tDCS. The parameters of active tDCS included $2 \mathrm{~mA}$ for 20 minutes per day for 10 consecutive days, anode over the left DLPFC (F3), cathode over the right DLPFC (F4) region.

Results. After 10 sessions of anodal and cathodal tDCS, patients showed significantly improved performance in visual and spatial aspects of memory tasks. Specifically, anodal stimulation improved visual memory performance for the experimental group relative to baseline, whereas sham stimulation did not differentiate performance from baseline in the control group.

Conclusion. This study showed that anodal tDCS over DLPFC concurrently with cathodal tDCS over right DLPFC improved visual and spatial aspects of memory in patients with MD. This finding is in generally consistent with previous findings about effectiveness of tDCS on cognition in MD, while additionally provides support for effectiveness of tDCS on visual memory in MD.

\section{ORAL PRESENTATIONS}

\section{Successful Treatment of 25 Consecutive Clients with Post-Concussion Syndrome using Neurofeedback and a Variety of Adjunctive Interventions}

Angelo Bolea, PhD, and Dennis Romig, PhD

Brain Injury Center, Maryland, USA

In 2013, the National Football League agreed to a $\$ 765$ million settlement to pay for professional football player post-concussion brain injuries. The recognition that all head 
injuries can have delayed-sometimes decades of delay-impact on cognitive, emotional, and behavioral functioning. Head injuries from all sports, traffic accidents, and military service are being more closely scrutinized for the delayed symptoms of post-concussion syndrome disabilities.

This presentation will offer proven assessment and treatment methods for post-concussion syndrome and other head injuries. It will apply the Quadrant Brain Theory of individualized Brain Quadrant EEG assessment and other neuropsychology assessments as the basis for treatment. Post-Concussion Syndrome and most head injuries are not a unitary condition, but instead are multifaceted with various constellations of symptoms. This necessitates careful assessment protocols that are linked with specific treatment methods that address the symptoms and clusters.

The results of a study of 25 consecutive clients with Post-Concussion Syndrome brain injuries will be presented. The application of the Quadrant Brain Theory was previously demonstrated as successful in the neurofeedback treatment of chronic inpatient schizophrenia (Bolea, 2010) and severe, suicidal depressed outpatient clients (Bolea \& Romig, 2013).

Quadrant Brain Theory-based neurofeedback was expanded with the post-concussion clients to include adjunctive modalities of intervention: respiration biofeedback, exercise, nutrition, sleep hygiene practices, and other behavioral and cognitive methods. The diagnostic characteristics of the clients will be described including the number of head injuries, the presence of psychological trauma, the variety of symptoms at intake, and the length of time between head injuries and onset of symptoms.

Of the 25 clients, 21 were completely rehabilitated, while the other 4 clients went from total disability to only partial disability. The neurofeedback protocols and the adjunctive treatments that were found most useful will be presented. The principles for which adjunctive modalities to use based upon the severity and co-morbidity of symptoms will be outlined.

\title{
References
}

Bolea, A. (2010). Neurofeedback treatment of chronic inpatient schizophrenia. Journal of Neurotherapy, 14(1), 47-54. http://dx.doi.org/10.1080/10874200903543971

\section{The Effects of Neurofeedback in the Default Mode Network: Pilot Study Results of Medicated Children with ADHD}

\author{
Lori Russell-Chapin, $P h D^{1}$, Ted Chapin, $P h D^{2}$, and Douglas Dailey, $L A c^{3}$ \\ ${ }^{1}$ Bradley University, Illinois, USA \\ ${ }^{2}$ Resource Management Services and the Neurotherapy Institute of Central Illinois, USA \\ ${ }^{3} \mathrm{~A}$ Matter of Mind, California, USA
}

Children with attention-deficit/hyperactivity disorder (ADHD) have difficulty activating the Default Mode Network (DMN) in a resting or quiet state. The DMN function assists in processing and understanding a person's internal, reflective world and the world of self and others. Neurofeedback (NFB), a type of EEG operant and classical conditioning, trains selfregulation skills using a brain-computer interface. The hardware and software have audiovideo capabilities to correct irregular brainwave patterns and regional cerebral blood flow 
associated with mental health and cognitive concerns. Individual treatment sessions usually last 20 minutes to gain the largest overall treatment effect. Typically NFB users need to experience about 30 to 40 sessions.

This experimental pre-post test design pilot study randomly assigned 12 children diagnosed with ADHD and currently on a stimulant medication to a treatment and control group.

Subjects in the treatment group completed 40 NFB sessions. Pre- and posttest fMRIs were administered on the treatment and control groups. Evidence showed that forty 20-minute sessions of Sensory Motor Rhythm (SMR) NFB consolidated the DMN allowing for appropriate activation in the posterior cingulate, precuneous, the temporoparietal junction and the cerebellar tonsils. In addition to regulating and increasing SMR at 12-14 Hz, our research results showed activation of the DMN in a resting state after 40 NFB sessions. Symptom checklists were completed after each NFB session, and our results showed significant overall symptom reduction. Our study showed that NFB treatment of medicated ADHD subjects led to clinical improvement that was accompanied by improvement in the DMN functions. Of additional interest is the group average pretreatment fMRI. This image suggests the limited anterior to posterior connectivity and the ectopic DMN components that are seen in the 9- to 15-year-old brain. The absence of these findings posttreatment suggests that the NFB also resulted in maturation of the DMN toward that of the adult brain in a period of several months.

Assisting children with ADHD to appropriately activate the DMN may help them be more adaptive and reflective and to better understand their own internal world and the world of others. A discussion of these research results and the maturation of the DMN will be at the core of this workshop.

The limitation of this study was the small sample size and male to female ratio of subjects. To address this concern, a large PCORI grant has been written and submitted to conduct further research on the effectiveness of NFB and the DMN with a sample size of 75 children with ADHD. A sham control has also been added.

\section{LORETA Z-Score Neurofeedback is Effective and Specific in the Treatment of Post- Traumatic Stress Disorder and Traumatic Brain Injury in Combat Veterans}

Dale Foster, $P h D$

Memphis Integral Neurofeedback Institute, Tennessee, USA

Post-traumatic stress disorder (PTSD) is becoming understood as a set of dynamic functional neural network disturbances through the advancement of increasingly accurate and available neuroimaging techniques. These same techniques are contributing to the understanding of traumatic brain injury (TBI) and the mechanics of both the common characteristics of mild $\mathrm{TBI}(\mathrm{mTBI})$ and the possible idiosyncratic neural disruptions. Since both PTSD and TBI can involve a wide range of neural dysregulations, maximal treatment outcome will result from optimal specificity of assessment and treatment. Combat veterans often suffer from both PTSD and $\mathrm{mTBI}$ resulting in numerous, complex, difficult to treat, and often disabling symptoms. This study reports on an ongoing project providing treatment to U.S. combat veterans suffering from both PTSD and mTBI utilizing 3-D tomographic EEG (tEEG) Low Resolution Brain Electromagnetic Tomography (LORETA) Z-Score Neurofeedback (LZN) driven by a symptom check list, functional neural network match (SCL-FNM) method. Objective and subjective results of the first 16 cases indicate this method is effective and 
specific. Subjective responses to the training indicate relatively rapid remediation of symptoms in many cases. Quantitative analyses using paired $t$-tests demonstrate significant normalization of current density and network connectivity measures in the targeted cortical and subcortical regions of training. Large effect sizes, based on Cohen's analysis, are observed in current density, phase, coherence, phase shift, and phase lock. A negative correlation between effect size and psychotropic medication was found along with a trend toward requiring less medication as training progressed. These interactions between LZN and psychotropic drugs provide a rationale for optimal cooperation among the trainee, LZN trainer, and prescribing physicians to maximize treatment efficacy. LZN based on the SCLFNM method is evidently both effective and specific in the treatment of combat veterans with PTSD and $\mathrm{mTBI}$. Based on the content of this presentation, the participant will be able to identify functional neural network models of PTSD and $\mathrm{mTBI}$ and understand and justify the use of LZN in the treatment of these disorders through the operant conditioning of current density, phase, coherence, phase lock, and phase shift.

\title{
Unlocking Parkinsonian Resting Tremor with Neurofeedback
}

\author{
Lisa Tataryn, MSc \\ Center for Neurofeedback, California, USA
}

Parkinson's disease is the second most common progressive degenerative disease affecting as many as one million individuals in the US and four million worldwide. Approximately four percent of people with Parkinson's are diagnosed before the age of 50 and the incidence increases with age. Currently there is no cure for Parkinson's disease and finding the right treatment as symptoms change over time is important and requires the expertise of specialized health care professionals.

Resting tremor, the most common symptom of Parkinson's disease, is treated with dopamine replacement therapy, surgical ablation, or with electrode implantation called Deep Brain stimulation. Innovative studies have discovered that tremor-correlated cortical activity can be detected by electroencephalography (Hellwig et al., 2000; Muthuraman, Raethjen, Hellriegel, Deuschl, \& Heute, 2008). The findings underline that the motor areas of the cerebral cortex are involved in the neuronal network generating resting tremor in Parkinson's disease. Further studies have confirmed the pathological neural network for Parkinsonian resting tremor (Brittain, Sharott, \& Brown, 2014; Brown, 2003; Helmich, Hallett, Deuschl, Toni, \& Bloem, 2012; Timmerman et al., 2003; Timmerman \& Fink, 2011). Understanding the cortical representation and localization of tremors makes it possible to develop Neurofeedback training protocols to decrease resting tremor.

This presentation will review scientific literature outlining the pathological network activity of Parkinsonian resting tremor. Case studies will be presented to demonstrate the decrease of Parkinsonian rest tremor during Neurofeedback training. The resting tremor is measured by Digitrac, a triaxial accelerometer that calculates and displays movement frequency and amplitude using Fast Fourier Transform (FFT). The tremor frequency and its three harmonics will be discussed. One case study of sham Neurofeedback for resting tremor with accelerometer recordings will be presented and discussed. Accelerometer recordings will also be provided to show the starting and stopping of rest tremors in a single session. Easily accessible tools for measuring resting tremor will be provided so that a clinician can monitor progress within a session and between sessions. 


\section{References}

Brittain, J. S., Sharott, A., \& Brown, P. (2014). The highs and lows of beta activity in corticobasal ganglia loops. European Journal of Neuroscience, 39(11), 1951-1959. http://dx.doi.org/10.1111/ejn.12574

Brown, P., (2003). Oscillatory nature of human basal ganglia activity; Relationship to pathophysiology of Parkinson's disease. Movement Disorders, 18(4), 357-363. http://dx.doi.org/10.1002/mds. 10358

Hellwig, B., Haubler, S., Lauk, M., Guschlbauer, B., Koster, B., Kristeva-Feige, R., ... Lucking, C. H. (2000). Tremor-correlated cortical activity detected by electroencephalography. Clinical Neurophysiology, 111(5), 806-809. http://dx.doi.org/10.1016/S13882457(00)00248-0

Helmich, R. C., Hallett, M., Deuschl, G., Toni, I., \& Bloem, B. R. (2012). Cerebral causes and consequences of parkinsonian resting tremor: A tale of two circuits? Brain, 135(11), 3206-3226. http://dx.doi.org/10.1093/brain/aws023

Muthuraman, M., Raethjen, J., Hellriegel, H., Deuschl, G., \& Heute, U. (2008). Imaging coherent sources of tremor related EEG activity in patients with Parkinson's disease. 30th Annual International Conference of the IEEE, Engineering in Medicine and Biology Society, Vancouver, British Columbia, Canada. http://dx.doi.org/10.1109/IEMBS.2008.4650266

Timmerman, L., Gross, J., Dirks, M., Volkmann, J., Freund, H.J., Schnitzler, A. (2003). The cerebral oscillatory network of parkinsonian resting tremor. Brain, 126(1), 199-212. http://dx.doi.org/10.1093/brain/awg022

Timmerman, L., \& Fink, G. R. (2011). Pathological network activity in Parkinson's disease: from neural activity and connectivity to causality. Brain, 134(2), 332-334. http://dx.doi.org/10.1093/brain/awq381

\section{Brain SPECT Scans, What is it, How May it Be Used to Help My Patients in My Practice}

Paul Wand, MD

Brain Healing Center of America, Pennsylvania, USA

A brain single-photon emission computerized tomography (SPECT) scan is a nuclear diagnostic test that provides information about the circulation of the brain and, to a lesser extent, the anatomy of the brain. The scan is performed by injecting a radioisotope intravenously and placing a camera around the patient's head. Radioactivity exits the patient's head and is absorbed by a special camera, and then that data is transferred into a computer. The computer has a program that converts the radioactivity into pictures of the brain similar to a MRI, as slices in both 2-D slices (axial, coronal, and sagittal) and 3-D surface maps unique to SPECT technology. The 3-D images are extremely useful in assessing trauma cases where the maximum injury is, to anterior poles of frontal and temporal lobes, which are easily imaged unlike in MRI, CT, or PET scans. Other advantages are that SPECT scans provide metabolic information about the circulation, and the results can be abnormal even though the structural imaging tests such as CT/MRI/PET are 
completely normal. Hence the notion of normal brain anatomy yet abnormal metabolic function is firmly established, and analogous to the well-documented example of an abnormal EEG and normal CT/MRI/PET of the brain. Another major clinical advantage is that it can provide the basis for a medical treatment in a variety of common clinical conditions, such as traumatic brain injury, ADD/ADHD, autism, and more. A common finding in many of the conditions listed above is a reduction of blood flow known as hypoperfusion. Since the brain is such a highly active metabolic organ, a very small degree of hypoperfusion can have a very deleterious consequence. Thus any treatment that either increases blood flow and/or increases oxygen content in the blood/brain will improve brain function. A medication with the specific purpose of increasing cerebral blood exists, and has been found to improve clinical symptoms in the conditions listed above. It is a safe FDA-approved drug in the category of calcium channel blockers, and is very well tolerated with minimal or no side effects. Hyperbaric oxygen has a similar beneficial effect on improving function of the existing population of neurons that were ischemic secondary to the cerebral hypoperfusion.

\section{Comprehensive Neuro-Programming in a Residential Context}

Stephen Barnard, MSW

CooperRiis Healing Community, Asheville, North Carolina, USA

In February 2014, the Cooper Riis Healing Community, a non-profit mental health facility in Asheville, NC, began offering its residents the opportunity to participate in what may be the most comprehensive neuromodulation and assessment program offered anywhere in the world within the context of a residential or inpatient setting. On-site qEEG assessments (using Deymed hardware and Neuroguide software) and computerized neuropsychological testing, various forms of neurofeedback (including Neuroguide LORETA and 19-channel Surface Z-score Training, Neuroguide Brainsurfer, EEG-driven audio-visual stimulation combined with neurofeedback, TAG Synchrony training, standard neurofeedback using Bio explorer and Deymed software and nirHEG), several different biofeedback modalities, as well as neurostimulation approaches such as CES, tDCS and AVS, are all part of the CooperRiis Neuroenhancement Program (CNEP). In addition, residents are encouraged to use BrainHQ computerized cognitive training exercises as well as to receive instruction in breathing techniques and mindfulness practices. Participants typically start with between 2 and 4 neurofeedback training sessions per week, in addition to ongoing opportunities to use CES, BrainHQ, and personalized breathing and mindfulness practice.

Our hypothesis is that this approach, offered within the context of a holistic program that encourages good nutrition, exercise, community involvement, as well as "standard" individual and group psychotherapeutic approaches, is uniquely suited to promote rapid healing, because positive neuroplastic change is encouraged in a comprehensive and integrated manner. We will examine the role a neuromodulation program plays within an already progressive model, how we promote it and educate other clinicians about its utility. Moreover, the unique advantages and challenges of being an integral part of a larger treatment team including psychiatrists, therapists, social workers, nutritionists, wellness coaches, and others, will be explored. We will discuss how we conceptualize utilizing nutrition to optimize brain training.

We will explain how we use each of the neuromodulation modalities in conjunction with each other, how we decide what to use, and how we monitor progress. We will discuss our impressions of the use of LORETA and surface z-score training combined with more 
"traditional" neurofeedback protocols, and how they can be used complementarily. Finally, we will present brief case summaries and an overview of both the clinical successes and failures to date. Specifically, we plan to present a case of possibly undiagnosed TBI and how we used many of the approaches mentioned above (HEG, qEEG-informed use of tDCS, CES, "standard" qEEG-informed bioexplorer neurofeedback, HRV training and BrainHQ training) and achieved remarkable results (as measured by follow-up qEEG and neuropsychological testing) in 4 weeks of training. We also hope to present cases featuring the combined use of qEEG-informed neurofeedback and tDCS to help with the challenges inherent in thought disorders such as schizophrenia.

\title{
Connectivity in Adult Attention-Deficit/Hyperactivity Disorder
}

\author{
Sarah Wyckoff, $P h D^{1}$, and Robert Coben, $P h D^{2}$ \\ ${ }^{1}$ Integrated Neuroscience Services/Sense Labs, Arizona, USA \\ ${ }^{2}$ Neurorehabilitation and Neuropsychological Services/Integrated Neuroscience Services, Arkansas, \\ USA
}

Objectives. Attention-deficit/hyperactivity disorder (ADHD) is a neurodevelopmental disorder characterized by symptoms of inattention, impulsivity, and hyperactivity that are observed across the lifespan. Despite the misconception that most children with ADHD mature out of the disorder, 30-65\% take their symptoms into adulthood (Faraone,

Biederman, \& Mick, 2006). Deviant electroencephalographic (EEG) patterns of activity have been repeatedly observed in adults with ADHD during resting-state conditions (Bresnahan \& Barry, 2002; Bresnahan, Barry, Clarke, \& Johnstone et al., 2006; Clarke et al., 2008; Hermens et al., 2004; Koehler et al, 2009; Loo et al., 2009; Woltering, Jung, Liu, \& Tannock, 2012). However, the investigation of coherence and connectivity differences in adult populations is limited. Clarke et al. (2008) was the first to investigate pairwise coherence differences among male adults with ADHD and age- and gender-matched controls, during an eyes-closed condition. The study findings revealed reduced hemispheric differences in the delta band at long inter-electrode distances and reduced alpha coherence at short-medium inter-electrode distances among ADHD participants. Further research is needed to replicate these findings and address the limitations of the initial study. In addition to addressing the issues of a small sample size, lack of female participants, and lack of an eyes-open condition, the current study will also investigate the known limitations of using pairwise coherence measurements (for review see, Coben, Mohammad-Rezazadeh, \& Cannon, 2014).

Methods. Continuous 21-channel EEG, in accordance with the International 10-20 System, was acquired from 46 adult participants (27 male, male-to-female ratio, 1.4:1.0) with DSM-IV defined ADHD and 46 age- and gender-matched healthy controls. For each frequency band (delta, theta, alpha, beta) and condition (eyes-closed and eyes-open), a mixed ANOVA was used to examine between group differences in coherence calculated with pairwise (per Clarke et al., 2008) and multivariate (Coben et al., 2014) approaches. Partial correlation coefficients were calculated between ADHD behavioral measures and coherence data.

Results. This investigation is currently in progress. The most current data will be presented at the time of the ISNR conferences.

Conclusion. This study is the first to investigate EEG coherence differences in a mixed gender adult ADHD sample during eyes-closed and eyes-open conditions using pairwise and 


\section{NeuroRegulation}

multivariate coherence estimates. Treatment implications, study limitations, and future directions in research will be addressed.

\section{References}

Bresnahan, S. M., \& Barry, R. J. (2002). Specificity of quantitative EEG analysis in adults with attention deficit hyperactivity disorder. Psychiatry Research, 112(2), 133-144. http://dx.doi.org/10.1016/S0165-1781(02)00190-7

Bresnahan, S. M., Barry, R. J., Clarke, A. R., \& Johnstone, S. J. (2006). Quantitative EEG analysis in dexamphetamine-responsive adults with attention deficit/hyperactivity disorder. Psychiatry Research, 141(2), 151-159. http://dx.doi.org/10.1016/j.psychres.2005.09.002

Clarke, A. R., Barry, R. J., Heaven, P. C. L., McCarthy, R., Selikowitz, M., \& Byrne, M. K. (2008). EEG in adults with attention-deficit/hyperactivity disorder. International Journal of Psychophysiology, 70(3), 176-183. http://dx.doi.org/10.1016/j.ijpsycho.2008.07.001

Coben, R., Mohammad-Rezazadeh, I., \& Cannon, R. L. (2014). Using quantitative and analytic EEG methods in the understanding of connectivity in autism spectrum disorders: a theory of mixed over- and under-connectivity. Frontiers in Human Neuroscience, 8(45), 1-12. http://dx.doi.org/10.3389/fnhum.2014.00045

Faraone, S. V., Biederman, J., \& Mick, E. (2006). The age-dependent decline of attention deficit hyperactivity disorder: a meta-analysis of follow-up studies. Psychological Medicine, 36(2), 159-165. http://dx.doi.org/10.1017/S003329170500471X

Hermens, D. F., Williams, L. M., Lazzaro, I., Whitmont, S., Melkonian, D., \& Gordon, E. (2004). Sex differences in adult ADHD: a double dissociation in brain activity and autonomic arousal. Biological Psychology, 66(3), 221-233.

http://dx.doi.org/10.1016/j.biopsycho.2003.10.006

Koehler, S., Lauer, P., Schreppel, T., Jacob, C., Heine, M., Boreatti-Hümmer, A., ... Herrmann, M. J. (2009). Increased EEG power density in alpha and theta bands in adult ADHD patients. Journal of Neural Transmission, 116(1), 97-104. http://dx.doi.org/10.1007/s00702-008-0157-x

Loo, S. K., Hale, T. S., Macion, J., Hanada, G., McGough, J. J., McCracken, J. T., \& Smalley, S. L. (2009). Cortical activity patterns in ADHD during arousal, activation, and sustained attention. Neuropsychologia, 47(10), 2114-2119. http://dx.doi.org/10.1016/j.neuropsychologia.2009.04.013.

Woltering, S., Jung, J., Liu, Z., \& Tannock, R. (2012). Resting state EEG oscillatory power differences in ADHD college students and their peers. Behavioral and Brain Functions, 8(60), 1-9. http://dx.doi.org/10.1186/1744-9081-8-60 


\section{Accelerated Recovery from Traumatic Brain Injury (TBI) with Z-score Neurofeedback Therapy}

J. Lucas Koberda, MD, PhD, Andrew Moses, BA, Jessica Winslow, BA, Paula Koberda, Laura Koberda, BA, and Andrew Bienkiewicz, BA

Tallahassee NeuroBalance Center, Florida, USA

Multi-case study. Forty patients diagnosed with mild to severe TBI were subjected to Zscore LORETA neurofeedback (NFB) therapy in our practice since 2011. Most of patients were diagnosed with mild $\mathrm{TBI}(\mathrm{mTBI})$ and were treated within the first year after their brain injury. Few patients were diagnosed with more severe TBI and were subjected to delayed outpatient NFB therapy (more than one year since their TBI). Most of the patients complained of headaches and cognitive problems while some also suffered from dizziness and overlapping depression. Those who complained of cognitive problems were subjected to analysis with computerized cognitive testing (Neurotrax, Inc) before and after 10 sessions of NFB. During NFB therapy a subjective response from patients was collected to discern whether or not there was an improvement of symptoms. In addition, QEEG maps were obtained before each NFB session initiation in order to see an objective improvement of QEEG abnormalities. Subsequent analysis revealed that all patients $(100 \%)$ noticed subjective improvement of their symptoms during 10 sessions of NFB therapy, out of which most of them reported initial improvement after only 1 to 3 sessions. Thirty-five patients also had an objective improvement $(87 \%)$ of QEEG maps manifesting as reduction of excessive beta activity and/or normalization of delta or theta power. Fifteen patients completed preand post-NFB cognitive testing with 14 patients (93\%) having significant cognitive enhancement (Global Cognitive Score increased 3-23 points with 12 points on average) after 10 sessions of NFB therapy. When compared to another cognitive enhancement group from our practice (consisting mostly of ADD patients) the TBI group resulted in even greater cognitive recovery. These results are very encouraging and indicate high potential of Z-score LORETA NFB in rehabilitation of patients suffering from TBI.

\section{Rocking the Brain: Bi-directional Training as a Strategy to Facilitate Progress in Neurofeedback Training}

Peter Van Deusen, MHA

The Learning Curve, Inc., Virginia, USA

Brain training traditionally has focused heavily on training to inhibit or reward amplitude at certain frequencies or level of synchrony between two sites at a frequency. Our experience with multiple-clinician supervision groups is that certain types of such training (e.g., downtraining eyes-open alpha or high-coherence fast frequency activity) can be extremely difficult for the client to achieve. Resistance to releasing an established pattern can slow or even block results.

Nearly two decades ago we discovered, somewhat by accident, that training such a pattern in the "wrong" direction first, then reversing the direction of training-as one would "rock" a stuck automobile to get out of a snowdrift or mud-can have a very positive effect.

During the last 2 years we have implemented "rocking" approaches to training amplitude, synchrony, and nIR HEG with impressive results. 
1. Training designs for coherence work-two- and four-channel-allow the trainer to shift from increasing to reducing coherence at the click of a button in the software.

2. Training designs for amplitude also allow the trainer to switch direction in a matter of moments.

3. The LIFE game, developed for use with nIR HEG training, actually trains the client to increase blood supply_-then reduce it—in the PFC.

It appears that many brains move more easily in a direction to which they are already committed. Challenging a brain that is already excessively locked in a beta frequency to lock tighter is less threatening than asking it to unlock. When, after 2 minutes, the brain is asked to let go and move back to the state it normally maintains, it tends to do so easily and often "overshoots" on the low side. After 2 minutes of this approach, we reverse again, and push the brain back up for 2 minutes, then again nudge it back down. This, in a sense, builds a "momentum" that frequently breaks the longstanding pattern.

We have discovered that the same approach used in amplitude training (e.g., training UP theta when it is already high before reducing it) also has a positive effect-especially in the first 1 to 3 sessions.

The LIFE nIR HEG game provides feedback for alternating between single-pointed focus (maximizing oxygenated blood flow) and open-focus states of pure consciousness. The client is trained in the first 2 to 4 sessions to learn to apply each state for a full session, and then he is trained to shift back and forth.

One of the great benefits of this approach has been the ability to use nIR HEG with OCD, anxiety, and other states where executive function may be excessive. It has also had the positive effect of minimizing over-training effects, allowing clients to train generally a minute longer per site than they normally would because of the "warm-down" period that follows the intense focus. We teach the client the same techniques we would use with HRV for shifting from closed to open focus.

\title{
The Use of a Neurofeedback Intervention in the Remediation of Neurophysiological Impairment Following Chemotherapy in Breast Cancer Patients
}

\author{
Stephen Sideroff, $P h D^{1}$, David Wellisch, $P h D^{1}$, David Kaiser, $P h D^{2}$, and Barry Kendall, \\ PsyD $D^{3}$ \\ ${ }^{1}$ Department of Psychiatry; University of California, Los Angeles, California, USA \\ ${ }^{2}$ SKIL (Sterman Kaiser Imaging Laboratory), New York, USA \\ ${ }^{3}$ Private Practice, California, USA
}

Cognitive impairment-including declines in attention, learning, memory, and processing speed-is reported by as many as $50 \%$ to $70 \%$ of patients experiencing cancer chemotherapy (Dietrich, Monje, Wefel, \& Meyers, 2008; Vardy \& Tannock, 2007). This impairment does not appear to remediate over time (Koppelmans et al., 2012) and has no effective treatment. While neuroimaging has revealed organic damage (Saykin et al., 2003; Stemmer, Stears, Burton, Jones, \& Simon, 1994), neurophysiological impairment has not been shown. 
Neurofeedback has been shown to be helpful in improving cognitive performance (e.g., Monastra, Monastra, \& George, 2002; Nelson \& Esty, 2012). The present study was performed to determine what neurophysiological impairment can be shown in this population and if a neurofeedback intervention can normalize these impairments.

Nine breast cancer subjects, with significant cognitive impairment as demonstrated by neurocognitive testing, and at least 1-year post-chemotherapy, were given a neurofeedback training intervention. The intervention consisted of eighteen 30-minute sessions of neurofeedback at two training sites, C3 and C4 simultaneously and occurring three times per week. Pre- and post-training QEEGs were performed to determine initial neurophysiological functioning as well as improvement following training.

In examining the pre-training eyes closed EEG, we found that all nine subjects demonstrated abnormal spectral magnitude across five bands compared to healthy adults (SKIL normative database; $p<0.05$, using a Z-score comparison of the individual to the normative data base; $t$-distribution comparison). Bands were 2-4, 4-8, 8-13, 13-34, and 34-39 Hz, respectively. Excessive (two or more standard deviations from norm) delta activity was shown by 4 of the 9 , and 7 showed deficit theta activity. I n addition, 6 of the 9 also demonstrated excessive gamma activity.

We compared integer frequency bands (e.g., $2 \mathrm{~Hz}, 3 \mathrm{~Hz}, 4 \mathrm{~Hz}$ ) pre-training to post-training eyes-closed QEEG spectral magnitudes. All comparisons were between an individual and the database norm with two or more Z-score deviations from normative values indicating abnormality. Of the 9 subjects, 7 showed significant normalization of band activity, and the remaining 2 showed significant compensatory changes.

Average improvement across subjects was $40 \%$, meaning that $40 \%$ of the abnormal frequency bands were in normal range after training. Half of the individuals who normalized, normalized completely for the entire frequency band; whereas the other half normalized for half of the range of the band, on average. Of the 4 subjects with excessive delta wave activity prior to training, 3 normalized in delta activity; and of the 7 subjects with diminished theta activity, 3 normalized in theta activity. Out of the 6 subjects with gamma excesses, 2 significantly improved (normalized) but 2 showed more excessive gamma activity after training. Improvement of symptoms such as anxiety and sleep will also be presented. Positive neurocognitive testing results will be presented separately.

Conclusions. Pre-training baselines demonstrated significant neurophysiological impairment. This impairment, particularly elevated delta frequency band, is consistent with cognitive deficits. The neurofeedback intervention produced significant improvements in the direction of normalization of the EEG. Discussion will include implications of using a standardized neurofeedback protocol, and suggestions for study replication, facilitated by specific protocol parameters.

\section{References}

Dietrich, J., Monje, M., Wefel, J., \& Meyers, C. (2008). Clinical patterns and biological correlates of cognitive dysfunction associated with cancer therapy. The Oncologist, 13(12), 1285-1295. http://dx.doi.org/10.1634/theoncologist.2008-0130 
Koppelmans, V., Breteler, M. M. B., Boogerd, W., Seynaeve, C., Gundy, C., \& Schagen, S. B. (2012). Neuropsychological performance in survivors of breast cancer more than 20 years after adjuvant chemotherapy. Journal of Clinical Oncology, 30(10), 10801086. http://dx.doi.org/10.1200/JCO.2011.37.0189

Monastra, V. J., Monastra, D. M., \& George, S. (2002). The effects of stimulant therapy, EEG biofeedback, and parenting style on the primary symptoms of attentiondeficit/hyperactivity disorder. Applied Psychophysiology and Biofeedback, 27(4), 231-249. http://dx.doi.org/10.1023/A:1021018700609

Nelson, D. V., \& Esty, M. L. (2012). Neurotherapy of traumatic brain injury/posttraumatic stress symptoms in OEF/OIF veterans. Journal of Neuropsychiatry and Clinical Neurosciences, 24(2), 237-240. http://dx.doi.org/10.1176/appi.neuropsych.11020041

Saykin, A. J., Ahles, T. A., Schoenfeld, J. D., Wishart, H. A., Petras, C. G., Furstenburg, C. T., ... Mamourian, A. C. (2003). Grey matter reduction on voxel-based morphometry in chemotherapy-treated cancer survivors. Journal of the International Neuropsychological Society, 9(2), 246.

Stemmer, S. M., Stears, J. C., Burton, B. S., Jones, R. B., \& Simon, J. H. (1994). White matter changes in patients with breast cancer treated with high-dose chemotherapy and autologous bone marrow support. American Journal of Neuroradiology, 15(7), 1267-1273.

Vardy, J., \& Tannock, I. (2007). Cognitive function after chemotherapy in adults with solid tumours. Critical Reviews in Oncology/Hematology, 63(3), 183-202.

\section{Functional Connectivity, Diffusion Tensor Imaging (DTI) AND LORETA Coherence, Phase and Co-Modulation}

Robert Thatcher, PhD

Applied Neuroscience Research Institute, Florida, USA

Objectives. The purpose of this study was to explore the relationship between diffusion tensor imaging (DTI) measured cortico-cortical ' $U$ ' shaped fibers and association fasciculi and electroencephalogram (EEG) source localization using LORETA. Another objective was to link the Talaraich Atlas spacings measured using DTI and the observed spacings between maxima and minima of cross-correlations and coherence of LORETA derived EEG time series.

Methods. The EEG was recorded from 19 scalp locations from 71 subjects ranging in age from 13 years to 16 years in the eyes-open and eyes-closed conditions. LORETA time series from 88 Brodmann areas was computed. Coherence between the within hemisphere Brodmann areas and homologous Brodmann areas was computed with special emphasis on phase differences that demonstrated network functional connectivity and not volume conduction. Similarly, the Pearson product correlation was computed between current density produced by Brodmann areas located within each hemisphere as well as hemispheric homologous Brodmann areas. Spatial heterogeneity between Brodmann areas cannot be 
accounted for based on volume conduction and the comparative consistency between coherence and co-modulation of current sources was evaluated.

Results. An spatial pattern of increasing and decreasing coherence and correlations were present with a spacing of approximately $2-3 \mathrm{~cm}$ in registration with the ' $U$ ' shaped fibers as measured by DTI (Catani \& de Shotten, 2012). Co-registration with longer distant cortical fasciculi were also present, especially in the frontal-occipital and frontal-temporal fasciculi. The presence of long phase differences and spatial heterogeneity that corresponds to the DTI Atlas heterogeneity shows that the results are due to network connectivity and not volume conduction.

Conclusions. There is reasonable cross-validation of the spatial distance between LORETA functional connectivity and the ' $U$ ' shaped fibers and association fasciculi. This is important because it emphasizes the underlying covariance between the wiring diagrams of the brain and the EEG sources that are dependent on cortico-cortical connections between Brodmann areas. DTI fiber connection location and heterogeneity is gives rise to different conduction velocities and different densities of connections between Brodmann areas. The linkage of the underlying wiring patterns of the brain by DTI and EEG LORETA provides a richer view of the EEG than if this linkage was not demonstrated. The objective is to learn how one can validate the spatial structure of the human EEG based on the density and distance of connections between Brodmann areas

\section{References}

Catani, M., \& de Shotten, M. T. (2012). Atlas of Human Brain Connections. Oxford, UK: Oxford University Press.

\section{Enhancing Your Practice to Work with Military Personnel}

Troy Todd, $P h D^{1}$, and David Hagedorn, $P h D^{2}$

${ }^{1}$ Personal Performance Solutions, Colorado, USA

${ }^{2}$ Evoke Neuroscience, North Carolina, USA

Because of the increased demands on the military since 11 September 2001, many of us have and will treat current and former military members, providing clinical and peak performance services. The military culture with its rapid deployment cycles, high potential for traumatic brain injury (TBI) and post-traumatic stress disorder (PTSD), unique family dynamics, and stressful work environments require all providers working with military members to be well informed to be effective. Without knowledge of these areas, the provider risks making errors that may alienate or even harm military members.

This presentation will help providers be more confident in working with military members by providing a practical understanding of the unique aspects of military culture and exposing them to clinical techniques that are effective in these populations.

The presentation will consist of two parts. In the first part, focus will be placed on the cultural aspects of working with the military, relevant to effective treatment of its members. Emphasis will be given to aspects of the military work environment, subtle but highly relevant stressors of deployment, unusual diagnostic considerations for military members, and the 
characteristics of family dynamics. During the second part, findings from EEG, ERPs, and ECG in PTSD and mTBI cases, as well the effective use of Brodmann area and surface amplitude neurofeedback with these patients will be discussed. Particular emphasis will be give on how the multifactorial nature of cognitive impairment and peak performance necessitates rapid, inexpensive, and easily applied multimodal analysis methods that can offer greater sensitivity and specificity for typical military cases. Case analysis will emphasize ease-of-use and accessibility for a clinical practice or peak performance program.

Case presentations, pictures from the deployed and "in garrison" military environments will be used to make this presentation applicable, and this often misunderstood portion of the population comprehensible, thus increasing competence in providers working with this population. Understand better how to work with military members in a clinical and peak performance practice settings. Deployment stressors and their effects.

\section{LORETA Neurofeedback in Precuneus for Substance Use Disorders}

Rex Cannon, PhD, Henry E. Pigott, PhD, Lisa Cicetti, PsyD, John DeGaglia, PhD, Susan Atlee, PhD, Jessica Heimburger, BA, Jared Neubrander, BA, and Christopher O'Brien, BA New Directions, LLC, Florida, USA

Introduction. Substance use disorders continue to exact substantial negative effects on society and the individual. This clinical study was conducted in an outpatient program. LORETA neurofeedback was utilized to target alpha current source density in a three-voxel cluster of neurons in the left precuneus. Patients completed between 3 and 5 sessions per week and 17 sessions total.

Methods. This study was conducted with 20 individuals, 10 male, mean age of 27.17 . All patients were diagnosed with a substance use disorder including heterogeneous comorbid syndromes including depression, anxiety and attention-deficit/hyperactivity disorder.

Outcomes were evaluated using neurophysiological and neuropsychological assessments. Clients were given pre-post testing assessments including the Integrated Visual and Auditory Continuous Performance Test (IVA+/CPT), Verbal Fluency and Color Word Interference Tasks from the Delis-Kaplan Executive Function System (DKEFS), Personality Assessment Inventory (PAI), Pittsburgh Sleep Quality Index (PSIQ) and a Brief Substance Craving Scale (BSCS).

Results. LORETA current source increased significantly at the region of training (ROT) as well as in specific nodes shown to be associated with reward and addiction (e.g., right insula, medial prefrontal, and parietal regions). The default and salience networks also show significant shifts in both CSD levels and connectivity. Attention and executive function scales improved significantly or in the desired direction post-training, with the clinical scales on the PAl showing dramatic and significant reductions. There was notable increase in sleep improvement and reductions in cravings.

Discussion. Data are still being collected and analyzed; however, perceptible post-training changes are expected to increase across sample size. Treatment of SUD shows mixed results concerning outcomes and pre- and posttreatment measures. LORETA neurofeedback may provide an important mechanism for treatment of SUD in conjunction with other therapeutic methods. The authors will discuss these complimentary methods in 
terms of self-perception, neural plasticity, and efficiency and the importance of brain-based treatment for addictive disorders.

\title{
Investigating Efficacy of 19-Channel Z-Score Neurofeedback in a Clinical Setting
}

\author{
Nancy Wigton, $P h D^{1}$, Genomary Krigbaum, $P s y D^{1}$, and Eugenia Bodenhamer-Davis, $P h D^{2}$ \\ ${ }^{1}$ Grand Canyon University, College of Doctoral Studies, Arizona, USA \\ ${ }^{2}$ University of North Texas, Department of Disability and Addictions Rehabilitation, Texas, USA
}

Objectives. In recent years new neurofeedback (NF) models have emerged based on the incorporation of live normative database derived z-scores, termed z-score NF (ZNF) (Collura, Thatcher, Smith, Lambos, \& Stark, 2009; Thatcher, 2012). Among these, the surface montage 19-channel z-score neurofeedback (19ZNF) is a new NF model. Yet, peerreview literature lacks research using quantitative-methodologies, with group means statistical analysis of outcome measures, to evaluate 19ZNF. Currently, case studies (Hallman, 2012; Koberda, Moses, Koberda, \& Koberda, 2012) and descriptive/clinical reviews (Thatcher, 2013; Wigton, 2013) make up the majority of peer-reviewed 19ZNF literature. The purpose of this quantitative study was to investigate the efficacy of 19ZNF, in a clinical setting, using archival data, with statistical analysis of group means data. This study also proposes an original method for measuring overall normalization of QEEG zscores suitable for group means research designs.

Methods. This study implemented a retrospective one-group pretest-posttest design, and used outcome measures of QEEG z-scores and the clinical assessments of the Integrated Visual and Auditory continuous performance test (IVA), the Behavior Rating Inventory of Executive Functioning (BRIEF), and the Devereux Scale of Mental Disorders (DSMD). Each outcome measure framed a group such that 19ZNF was evaluated as related to electrocortical functioning $(n=21)$, as well as the neuropsychological constructs of attention ( $n=10)$, executive function $(n=12)$, and behavior $(n=14)$. Thus, the four research questions were as follows: Does 19ZNF improve (1) electrocortical function as measured by QEEG z-scores, (2) attention as measured by the IVA, (3) executive function as measured by the BRIEF, and (4) behavior as measured by the DSMD? One-tailed $t$-tests were performed to compare the pre-post scores for the selected QEEG metrics and the included scales of the clinical assessments.

Results. The direction of change, for all pre-post comparisons, was in the predicted direction. For all clinical assessments and z-scores, the group means were beyond the clinically significant threshold before 19ZNF, and no longer clinically significant after 19ZNF. The pre- to post-difference for all scales/metrics for all outcome measures were statistically significant, with results ranging from $p=.000$ to $p=.008$; and effect sizes ranging from 1.29 to 3.42. In general, the number of session for all groups was approximately 10 (ranging from 9.70 to 11.83 sessions) at a session frequency of on average of once per week.

Conclusions. Results suggest 19ZNF improved electrocortical function, attention, executive function, and behavior. Moreover, these findings support 19ZNF as having potential to bring about both QEEG normalization and symptom improvement. While clearly more research is needed, this study provides beginning evidence of the efficacy of 19ZNF. Additionally, this study supports prior clinical reports (Thatcher, 2013; Wigton, 2013) that 19ZNF results in improvement of clinical symptoms in fewer sessions than traditional NF. In sum, this 
research adds to what is known about 19ZNF and offers a methodology for using QEEG metrics as outcome measures. Based on the content of this presentation, the participant will be able to understand the parameters of the efficacy of 19ZNF, within the context of this study, regarding how 19ZNF improved electrocortical function, attention, executive function, and behavior.

\section{References}

Collura, T. F., Thatcher, R. W., Smith, M. L., Lambos, W. A., \& Stark, C. R. (2009). EEG biofeedback training using live z-scores and a normative database. In T. H.

Budzynski, H. K. Budzynski, J. R. Evans \& A. Abarbanel (Eds.), Introduction to QEEG and neurofeedback: Advanced theory and applications. (2nd ed., pp. 103141). Burlington, MA: Elsevier.

Hallman, D. W. (2012). 19-Channel neurofeedback in an adolescent with FASD. Journal of Neurotherapy, 16(2), 150-154. http://dx.doi.org/10.1080/10874208.2012.677646

Koberda, J. L., Moses, A., Koberda, L., \& Koberda, P. (2012). Cognitive enhancement using 19-electrode z-score neurofeedback. Journal of Neurotherapy, 16(3), 224-230. http://dx.doi.org/10.1080/10874208.2012.705769

Thatcher, R. W. (2012). Handbook of Quantitative electroencephalography and EEG Biofeedback. St. Petersburg, FL: ANI Publishing.

Thatcher, R. W. (2013). Latest developments in live z-score training: Symptom check list, phase reset, and LORETA z-score biofeedback. Journal of Neurotherapy, 17(1), 6987. http://dx.doi.org/10.1080/10874208.2013.759032

Wigton, N. L. (2013). Clinical perspectives of 19-channel z-score neurofeedback: Benefits and limitations. Journal of Neurotherapy, 17(4), 259-264. http://dx.doi.org/10.1080/10874208.2013.847142

Wigton, N. L. (2014). Evaluating 19-channel z-score neurofeedback: Addressing efficacy in a clinical setting. (Doctoral dissertation). Available from ProQuest Central database (UMI No. 3625170).

\section{Training Attention, Working Memory, and Information Processing Speed Using 3D-MOT}

Brendan Parsons, BA, MSc Candidate, and Jocelyn Faubert, PhD Neurodezign / University of Montreal, Canada

3D-MOT is a perceptual-cognitive training system based on multiple object tracking (MOT) in a three-dimensional (3D) virtual environment. The current presentation will discuss 3D-MOT as an adjunct to neurofeedback intervention for healthy individuals looking to improve cognitive performance, individuals with attention deficits, individuals recuperating from head trauma, high-level athletes, and aging populations.

To this end, current research will be presented that shows that 3D-MOT training can enhance attention, working memory and visual information processing speed in healthy university 
students; correlates of brain functions are also assessed using quantitative electroencephalography (Parsons et al., in press). Further research demonstrates attention improvements in school-aged children with attention deficits (Parsons, Bates, \& Faubert, in press). Research currently underway has demonstrated the potential of using 3D-MOT has a rehabilitation tool for those suffering from head trauma (Romeas et al., in press). Research has also shown a strong link between athletic performance levels and learning capacity on this task (Faubert \& Sidebottom, 2012; Faubert, 2013). Finally, research has demonstrated transfer of this training to socially relevant tasks in aging populations (Legault \& Faubert, 2012; Legault, Allard, \& Faubert, 2013).

As a clinical tool, 3D-MOT offers relatively faster gains than neurofeedback; yet research into the longevity of training effects is currently lacking. Neurofeedback studies, however, have demonstrated the long-term retention of training effects. Combined, the two methods offer the best of both worlds: quick gains and long-term consolidation. A 3D-MOT session takes approximately 8 minutes to administer, and thus the length of a typical neurofeedback session would not have to be significantly lengthened.

Further, a novel research study used a hybrid, integrated EEG-based neurofeedback intervention and demonstrated enhanced cognitive functions similar to the study of Parsons and colleagues (in press) as well as augmented learning within the task in healthy university students (Parsons \& Faubert, in press).

\section{References}

Faubert, J., \& Sidebottom, L. (2012). Perceptual-cognitive training of athletes. Journal of Clinical Sports Psychology, 6(1), 85-102.

Faubert, J. (2013). Professional athletes have extraordinary skills for rapidly learning complex and neutral dynamic visual scenes. Scientific Reports, 3,1154. http://dx.doi.org/10.1038/srep01154

Legault, I., Allard, R., \& Faubert, J. (2013). Healthy older observers show equivalent perceptual-cognitive training benefits to young adults for multiple object tracking. Frontiers in Psychology, 4, 323. http://dx.doi.org/10.3389/fpsyg.2013.00323

Legault, I., \& Faubert, J. (2012). Perceptual-cognitive training improves biological motion perception: evidence for transferability of training in healthy aging. Neuroreport, 23(8), 469-473. http://dx.doi.org/10.1097/WNR.0b013e328353e48a

\section{The Combined Effects of Neurofeedback and Biofeedback Training for Treating Children with Autism Spectrum Disorder}

Matthew Goodman, BS, Rita Sharma, MSW, LSW, and Jaime Pineda, PhD

AIU/UCSD, California, USA

Autism Spectrum Disorder (ASD) is characterized by deficits in social communication and restricted, repetitive behaviors. Research suggests that the Mirror Neuron System (MNS), which is thought to mediate empathic behavior, may be dysfunctionally operating in this population, thus contributing to some of these deficits. Neurofeedback Training (NFT) has 
been able to improve MNS activity by targeting mu rhythms (8-13 Hz oscillations over the sensorimotor cortex). Peripheral physiological activity in ASD has received less research attention, yet also shows deficits and potential for clinical intervention. Heart Rate Variability (HRV) vagal activity is a key example of this. Moreover, these physiological mechanisms have been linked to social behavior and are believed to be highly integrated with central, neurological activity. An approach that targets this system as a whole could have a greater impact on behavior. This presentation will discuss a clinical intervention that uses a combination of NFT and HRV biofeedback to improve symptoms of autism. The study is currently taking place and is projected to be completed by September, 2014. Of the 24 participants, each will either receive (a) HRV Biofeedback only (Group 1), or (b) HRV Biofeedback and NFT (Group 2). Both groups will first learn how to diaphragmatic breathe, which is pivotal for conducting HRV Biofeedback. Then, one group will continue to receive HRV Biofeedback (through operant reinforcement of a DVD movie), while the other receives HRV Biofeedback and NFT (also through operant reinforcement of a DVD). It is hypothesized that Group 1 will show positive changes in HRV, vagal tone, and social behavior. It is hypothesized that Group 2 will show positive changes in HRV, vagal tone, mu suppression, long-range neural connectivity, and social behavior. Outcomes will be assessed through EEG and magnetoencephalographic (MEG) scans, physiological measurement (e.g., HRV and vagal tone), and behavioral questionnaires. This presentation aims to convey the basic neurological and physiological mechanisms thought to underlie autism and social behavior, and potential avenues to improve these mechanisms. Learn about the integration of biofeedback and neurofeedback.

\section{Insomnia in Returning Service Members an Early Intervention in Combat Stress}

Peter Smith, PsyD, and Glenda Wolfe, PMHNP

$1 / 1 \mathrm{EBH}$ William Beaumont Army Medical Center, Texas, USA

Introduction. Disrupted sleep, mood disorder and performance deficits have been observed among service members returning from combat. Insomnia is a crucial symptom in the posttraumatic stress disorder (PTSD) cluster (Maker, Rego, \& Asnis, 2006) and efforts to quickly address disrupted sleep in newly returning soldiers may enhance amelioration of the emergence of PTSD. EEG Biofeedback has been demonstrated to be of value in the treatment of PTSD symptoms (Peniston \& Kulkosky, 1991; Peniston, Marrinan, Deming, \& Kulkosky, 1993) but to date is not well established as a treatment option for active duty soldiers.

A program evaluation was designed to assess the effectiveness of insomnia focused $\mathrm{CBT} / \mathrm{CPT}$ group psychotherapy in conjunction with EEG (neurotherapy). This pilot study was designed to further evaluate the EEG/QEEG, Continuous Performance and self-report measures pre- and post-initial intervention at an Embedded Behavioral Health unit with newly returning combat Infantry soldiers.

Methods. Twenty-eight service members returning from deployment in 2013 with combat stress symptoms were recruited for a program evaluation as part of routine outpatient care at an Embedded Behavioral Health clinic at Fort Bliss, Texas. All service members in the evaluation received group psychotherapy in an intensive workshop format. The insomnia workshop consisted of four weekly 120-minute sessions. The initial two sessions of this workshop consisted of CBT Stimulus Pairing training and cognitive re-appraisals about 
insomnia. The remaining two sessions of this workshop focused on CBT methods to address nightmares in accordance with research (Davis \& Wright, 2005).

After completion of the workshops, service members could elect a course of 20 sessions of EEG assisted biofeedback. Those who elected this modality received an analog/QEEG (digital) 19-channel baseline with attentional testing at initiation of biofeedback along with standard clinic measures PCL-M, GAD, and PHQ pre- and post-EEG biofeedback treatment. Participants were assigned to 1 of 3 EEG Biofeedback systems that included either the Brainpaint, LENS (Low Energy Neurotherapy System), or a task activated EEG Biofeedback program developed by Marvin Sams, ND. All participants completed weekly sleep logs and reported changes in medication utilization and tobacco/alcohol use.

Results. An analysis of outcome measures was performed for all members who completed self-report inventories after 20 EEG biofeedback sessions. Data analysis of the IVA continuous performance measures demonstrated statistical significance on pre vs. post measures of the Full Scale Attention Quotient as well as the Visual Attention Quotient. All means scores of each of the IVA variables improved from the average pretest scores. Data analysis of the EEG QEEGs is currently underway, as is clinic follow-up on the treated service members and those that dropped out of treatment during the course of the evaluation.

Conclusions. Disrupted sleep is postulated as an early marker of combat related stress disorders (PTSD). The authors have sought a pilot program to assist with objective measures to enhance treatment outcomes in service members at risk to develop PTSD. It is hoped that this initial pilot study may provide a stimulus for further research that demonstrates that timely rapid and effective relief of symptoms will improve outcomes. Introduce treatment outcomes for interventions for Insomnia in recently redeployed combat soldiers.

\section{References}

Davis, J. L., \& Wright, D. C. (2005). Case series utilizing exposure, relaxation, and rescripting therapy: Impact on nightmares, sleep quality and psychological distress. Behavioral Sleep Medicine, 3(3), 151-157. http://dx.doi.org/10.1207/s15402010bsm0303_3

Maker, M. J., Rego, S. A., \& Asnis G. M. (2006). Sleep disturbances in patients with posttraumatic stress disorder: epidemiology, impact and approaches to management. CNS Drugs, 20(7), 567-590. http://dx.doi.org/10.2165/00023210-200620070-00003

Peniston, E. G., \& Kulkosky, P. J. (1991). Alpha-theta brainwave neuro-feedback for Vietnam veterans with combat-related post-traumatic stress disorder. Medical Psychotherapy, $4,47-60$.

Peniston, E. G., Marrinan, D. A., Deming, W. A., \& Kulkosky, P. J. (1993). EEG alpha-theta brainwave synchronization in Vietnam theater veterans with combat-related posttraumatic stress disorder and alcohol abuse. Advances in Medical Psychotherapy, 6, $37-50$. 


\title{
Gamma ERS/ERD Development Cycles of Abstractions in Math Learning
}

\author{
Alex Hyungkyu Kwon, PhD \\ Kyungsung University, Korea
}

The present study describes an event-related synchronization and desynchronization (ERS/ERD) in the gamma band $(31-50 \mathrm{~Hz})$ induced by the onset of abstractions in math learning stimuli. Three levels of tasks in abstractions are presented with spontaneous and comprehensions conditions in which abstract mappings (A2) with abstract systems (A3) and systems of abstract systems (A4) were held in math learning. The tasks are all based on the four mathematical operations of addition, subtraction, multiplication, and division. Dynamic growth models show the complex, interrelated changes that take place during brain growth, cognitive development, and learning (Fischer, Bernstein, \& Immordino-Yang, 2007). Math learning requires flexibility to adapt existing brain function in selecting new neurophysiological activities to learn desired knowledge. Flexibility exists over multiple brainwave scales as performance of a skill change from slow to fast and automatic learning. This study investigated the brainwave structure and connectivity by identifying dynamic changes of brain modularity. This research shows the complex network of interactions, convergent and divergent paths, nested cycles, stabilities and instabilities, progressions and regressions, clusters of discontinuities, and stable levels of performance (Fischer et al., 2007). It represents the relationships between brainwave activities and cycles of cognitive performance, which becomes most visible with optimal functioning of the cognitive system when the effective supports a student's performance. A series of discontinuities in optimal cognitive growth defines an abstract level scale. Brain development characteristics-such as number of neurons and synapses, brain mass, myelination, and brain activity-change by the cycles of cognitive performance. The brain changes by the simultaneous cognitive and emotional factors, in parallel with the individual difference and age. This study neurologically proves the framework of the dynamic systems theory for analyzing complex patterns of the spurts and drops of the development of abstractions. Neurologically proved complexity scale provides a useful ruler to track the individual differences in learning a specific task for math learning. The participant will be able to understand the event-related synchronization and desynchronization (ERS/ERD) in the gamma band $(31-50 \mathrm{~Hz})$ induced by the onset of abstractions in math learning stimuli.

\section{References}

Fischer, K. W., Bernstein, J. H., \& Immordino-Yang, M. H. (2007). Mind, brain, and education in reading disorders. Cambridge, UK; New York: Cambridge University Press.

\section{On the Relationship Between EEG Phase Reset in the Time Domain and EEG Power in the Frequency Domain}

Robert Thatcher, PhD

Applied Neuroscience Research Institute, Florida, USA

Objectives. The purpose of this study was to explore the relationship between EEG phase reset in the time domain and EEG absolute power in the frequency domain. Of special interest was how a synchronous group of neurons in one frequency band correlates with power in the same and different frequency bands. 
Methods. The electroencephalogram (EEG) was recorded from 19 scalp locations from 326 subjects ranging in age from 5 years to 17.6 years in the eyes open and eyes closed conditions. Complex demodulation was used to compute instantaneous EEG absolute power in the frequency domain (delta, theta, alpha, beta1, beta2, beta3 \& hi-beta). The first derivative of straightened phase differences for all electrode combinations for each frequency band and then phase shift duration and phase lock duration was computed between pairs of electrodes in the time domain. Correlations were then computed between EEG power and the duration of phase shift and phase lock in different frequency bands. Cross-frequency correlations were also computed to compare amplitude and phase reset relations across frequency bands.

Results. Positive correlations were present between phase shift and lock duration and absolute power in all frequency bands except the delta $(1-4 \mathrm{~Hz})$ frequency band. Inverse or competitive relationships were present between alpha1 $(8-10 \mathrm{~Hz})$ and alpha2 $(10-12 \mathrm{~Hz})$ and theta $(4-7 \mathrm{~Hz})$ frequency bands and between beta1 $(12-15 \mathrm{~Hz})$ and alpha $(8-12 \mathrm{~Hz})$ as well as between alpha1 $(8-10 \mathrm{~Hz})$ and alpha2 $(10-12 \mathrm{~Hz})$ frequency bands. Positive correlations were present between theta and beta1 frequency bands as well as between alpha2 and beta3 $(18-25 \mathrm{~Hz})$ frequency bands.

Conclusions. The spontaneous EEG is the summation of phase shifting and phase locking of competitive thalamocortical pacemaker systems. Cross-frequency coupling occurs by reciprocal inhibition of competing frequency resonance bands of synchronized groups of neurons. Learn how groups of neurons that synchronize in one frequency band affect EEG power in different frequency bands. Learn about cooperation and competition between groups of synchronous neurons in different frequency bands.

\section{QEEG and Neurofeedback for Elementary Students with ADHD Inattentive Subtype}

Michael Linden, $P h D^{1}$, and Jeff La Marca, $P h D^{2}$

${ }^{1}$ Attention Learning Center, California, USA

${ }^{2}$ University of California, Irvine, California, USA

Beginning with the first study on the use of neurofeedback (NF) as an intervention for attention-deficit disorder (ADD) and attention-deficit/hyperactivity disorder (ADHD; Lubar \& Shouse, 1976), improvements in school performance and positive outcomes on cognitive and academic measures have been consistently reported (Linden, Habib, \& Radojevic, 1996; Lubar, 1991; Thompson \& Thompson, 1998; Thornton, 2000). Research has demonstrated that NF is most efficacious for ameliorating symptoms of inattention (Arns, de Ridder, Strehl, Breteler, \& Coenen, 2009; Monastra, Monastra, \& George, 2002). Studies provide evidence that inattention is associated with reduced processing speed on school-related tasks (Weiler, Bernstein, Bellinger, \& Waber, 2000) and impaired processing of visual information (Barkley, Grodzinsky, \& DuPaul, 1992; Swanson, Posner, Potkin, \& Bonforte, 1991). Willcutt and Pennington (2000) found that the relationship between ADHD and reading disorders (RD) was stronger for students with symptoms of inattention than hyperactivity/impulsivity. A recent review of the literature (Sexton, Gelhorn, Bell, \& Classi, 2012) suggests that ADHD and RD are not just frequently associated comorbid conditions but are, indeed, co-occurring conditions that result in significant deleterious and chronic impairments that begin in childhood and extent into adulthood. 
The extant literature on NF now lends support that it is an efficacious intervention for ameliorating symptoms of ADHD (Arns et al., 2009; La Marca, 2011). This is evidenced by the recent decision of the American Academy of Pediatrics to state that the research on biofeedback (including neurofeedback) has met criteria to justify their highest level of support (Level 1: Best Support) as an evidenced-based psychosocial intervention for attention and hyperactivity (American Academy of Pediatrics, 2012). However, there remains a veritable dearth of research that specifically examines the use of NF to directly address academic achievement; the literature deals primarily with symptoms of attention.

This presentation will examine some of the findings of the first study to directly investigate the impact of NF on measures of reading achievement (La Marca, 2014). An examination of preand post-intervention QEEG and IVA CPT data will be presented. The role of theta/beta ratio reduction, particularly as it applies to addressing symptoms of inattention, will also be discussed.

In addition, we will review the history of using QEEG Ratio/Scans and QEEG Brain Mapping to assist in the diagnosis of ADHD and subtypes of ADHD. We will present different types of QEEG measures, such as theta/beta and theta/high beta ratios that differential inattentive ADHD from hyperactivity/impulsivity. Furthermore, we will present data that indicates that certain QEEG measures and ratios are more sensitive to the effects of neurofeedback on measures of attention and reading achievement.

\section{References}

American Academy of Pediatrics. (2012, October). Evidence-based child and adolescent psychosocial interventions. Retrieved from http://www.aap.org/en-us/advocacy-andpolicy/aaphealthinitiatives/MentalHealth/Documents/CRPsychosociallnterventions.pdf

Arns, M., de Ridder, S., Strehl, U., Breteler, M., \& Coenen, A. (2009). Efficacy of neurofeedback treatment in ADHD: The effects on inattention, impulsivity and hyperactivity: A meta-analysis. Clinical EEG and Neuroscience, 40(3), 180-189. http://dx.doi.org/10.1177/155005940904000311

Barkley, R. A., Grodzinsky, G., \& DuPaul, G. J. (1992). Frontal lobe functions in attention deficit disorder with and without hyperactivity: A review and research report. Journal of Abnormal Child Psychology, 20(2), 163-188.

La Marca, J. P. (2014). Neurofeedback as an intervention to improve reading achievement in students with attention deficit hyperactivity disorder, inattentive subtype (Doctoral dissertation). University of California, Riverside.

La Marca, J. P. (2011). Neurofeedback training for the enhancement of attention in ADD/ADHD children. Journal of Neurotherapy, 15(4), 437-438.

Linden, M., Habib, T., \& Radojevic, V. (1996). A controlled study of the effects of EEG biofeedback on cognition and behavior of children with attention deficit disorder and learning disabilities. Biofeedback and Self-Regulation, 21(1), 35-49. 


\section{NeuroRegulation}

Lubar, J. F. (1991). Discourse on the development of EEG diagnostics and biofeedback for attention-deficit/hyperactivity disorders. Biofeedback and Self-Regulation, 16(3), 201225.

Lubar, J. F., \& Shouse, M. N. (1976). EEG and behavioral changes in a hyperkinetic child concurrent with training of the sensorimotor rhythm (SMR). Applied

Psychophysiology and Biofeedback, 1(3), 293-306.

Monastra, V. J., Monastra, D. M., \& George, S. (2002). The effects of stimulant therapy, EEG biofeedback, and parenting style on the primary symptoms of attention-

deficit/hyperactivity disorder. Applied Psychophysiology and Biofeedback, 27(4), 231-249.

Swanson, J. M., Posner, M., Potkin, S. G., \& Bonforte, S. (1991). Activating tasks for the study of visual-spatial attention in ADHD children: A cognitive anatomic approach. Journal of Child Neurology, 6, S119-S127.

Thompson, L., \& Thompson, M. (1998). Neurofeedback combined with training in metacognitive strategies: effectiveness in students with ADD. Applied Psychophysiology and Biofeedback, 23(4), 243-263.

Thornton, K. (2000). Improvement/rehabilitation of memory functioning with neurotherapy/ QEEG biofeedback. Journal of Head Trauma Rehabilitation, 15(6), 1285-1296. http://dx.doi.org/10.1097/00001199-200012000-00008

Weiler, M., Bernstein, J., Bellinger, D., \& Waber, D. (2000). Processing speed in children with attention deficit/hyperactivity disorder, inattentive type. Child Neuropsychology, 6(3), 218-234. http://dx.doi.org/10.1076/chin.6.3.218.3156

Willcutt, E. G., \& Pennington, B. F. (2000). Comorbidity of reading disability and attentiondeficit/hyperactivity disorder: Differences by gender and subtype. Journal of Learning Disabilities, 33(2), 179-191. http://dx.doi.org/10.1177/002221940003300206

\section{Early Cognitive Decline and Alzheimer's Disease: Detection and Intervention Using sLORETA Z-scored Imaging}

Penijean Gracefire, MSMHC

Stress Therapy Solutions, Inc., Ohio, USA

This presentation will review the history of MRI research related to detecting early cognitive decline and Alzheimer's disease, compare and contrast MRI and SLORETA Z-scores as imaging techniques, and discuss potential applications of sLORETA Z-score imaging technology to both the categories of early detection and intervention with regard to cognitive decline and Alzheimer's disease in aging populations.

An increasing body of research on Alzheimer's disease and early cognitive decline using magnetic resonance imaging (MRI) has pinpointed the hippocampus and parahippocampal gyrus as the brain regions most likely to show functional abnormalities in the initial stages of cognitive decline. 
Early MRI studies demonstrated a significant deficit in the volume of the parahippocampal gyrus in subjects with aging-associated cognitive decline. Compared with cognitively intact subjects, subjects with aging-associated cognitive decline had a significantly smaller mean volume of the right parahippocampal gyrus. Additional research indicates the significant association between parahippocampal gyrus volumes and severity of cognitive deficits in aging-associated cognitive decline groups. This finding is in line with the view that pathological alterations in the parahippocampal gyrus, leading to a disconnection of the hippocampus from neocortical association areas, represent the neuroanatomical correlate of cognitive dysfunction in early dementia.

Consistent, although much more pronounced, changes were found in Alzheimer's disease patients, indicating that, with respect to parahippocampal gyrus volume, the subjects with aging-associated cognitive decline took an intermediate position between the Alzheimer's disease patients and the cognitively intact comparison subjects.

Neuropathological evidence supports the premise that the earliest changes in Alzheimer's disease selectively affect the parahippocampal regions of the brain. Studies conducted to determine if otherwise healthy elderly subjects with mild cognitive impairment had structural volume deficits affecting the parahippocampal gyrus resulted in findings indicating that parahippocampal atrophy underlies the observed cognitive deficits in aging-associated cognitive decline. These findings corroborate the hypothesis that aging-associated cognitive decline represents a preclinical stage of Alzheimer's disease.

Standardized low-resolution brain electromagnetic tomography (sLORETA) uses surface EEG readings to project a three-dimensional cortical image composed of 6,239 voxels approximately 5 millimeters in size. Each voxel is converted instantaneously into a current source density, and three spatial values (vectors) that describe the dipole. Thus, each voxel represents an "analog" number, the local level of activity. This is done in 16 frequency bands simultaneously, in real time.

All projected signals are time-domain signals, providing superior speed and accuracy compared to FFT-based approaches, which introduce delays and possible distortion, due to the epoch size limitations. FFT-based methods also show only averaged activity, not instantaneous values. The software computes the sLORETA projection instantaneously using high-speed time-domain methods, and accurately shows the momentary changes in EEG signals, in real time.

SLORETA has been extensively validated against MRI with regard to the accuracy of its localization methods, and can be used to not only evaluate parahippocampal activity relative to age-referenced normative values, but also to provide feedback to the brain based exclusively on activation patterns observed in the parahippocampal gyrus. Evaluate emerging trends in sLORETA imaging technology in the field of neurofeedback, with regard to applications potentially assessing and treating elderly populations exhibiting signs of early cognitive decline and preclinical Alzheimer's disease. 


\title{
Four-Channel Multivariate Coherence Training: Rationale and Findings
}

\author{
Robert Coben, $P h D$
}

Integrated Neuroscience Services, Arkansas, USA

Coherence training has been used as a form of neurofeedback for over 10 years. Empirical findings have shown it to be effective in the treatment or management of Traumatic Brain Injury (Walker, Norman, \& Weber, 2002), Autism Spectrum Disorder (Coben \& Padolsky, 2007), Learning Disabilities (Nazari, Mosanezhad, Hashemi, \& Jahan, 2012), and potentially many more conditions including Epilepsy (Coben, Wyckoff, \& Hudspeth, in press). However, all such coherence training is based on training coherence in pairs (sets of two electrode signals).

There is considerable research over the past decade to suggest that measuring coherence in pairs can lead to spurious errors and findings (Blinowska, 2011). Rather, there are forms of advanced multivariate statistical methods that can be used to calculate coherence that are more accurate and precise in depicting such relationships and even their directionality. In fact, one study has shown that using pairwise estimates can lead to up to $50 \%$ more errors than multivariate approaches. Given these findings, one wonders how this might impact neurofeedback training, as this has been the only way coherence training has ever been done. More so, could we develop another approach that would lead to even more effective treatments?

We developed a new methodology for performing coherence training that uses four channels of EEG data and bases calculations of coherence on multivariate methods. In this approach, four sensor locations are selected based on QEEG data and ongoing calculations are made such that each location is the average coherence between that electrode and the three others, and the same is done for each location. A summed coherence value is then derived that represents the average of all the averages and is considered to average multivariate coherence value of all channels. It is our belief that calculating and feeding back multivariate coherence estimates to the client will lead to more accurate information and enhance efficacy.

To test this hypothesis we designed a research program that has used this approach for more than 200 patients. Of these patients, more than 75 had also previously had twochannel coherence training and served as their own controls. We have then compared the effects of these trainings (two-channel coherence vs. four-channel multivariate coherence) on various clinical and EEG variables across multiple diagnoses. Preliminary analyses of a smaller cohort has already shown significant differences such that four-channel training leads to greater coherence and power changes in about half the number of sessions.

Our goal during this presentation is to present this methodology, study findings, and discuss the ramifications of how this can enhance efficacy in neurofeedback training. This large database also enables us to discuss how these issues interface with different diagnoses, clinical setting, gender, age, and many other variables. The participant will learn about a new form of neurofeedback and how it compares to more traditional two-channel coherence training. 


\section{References}

Blinowska, K. J. (2011). Review of the methods of determination of directed connectivity from multichannel data. Medical \& Biological Engineering \& Computing, 49(5), 521-529. http://dx.doi.org/10.1007/s11517-011-0739-x

Coben, R., \& Padolsky, I. (2007). Assessment-guided neurofeedback for autistic spectrum disorder. Journal of Neurotherapy, 11(1), 5-23. http://dx.doi.org/10.1300/J184v11n01_02

Coben, R., Wyckoff, S., \& Hudspeth, W. J. (in press). Impact of neurofeedback on epileptiform discharges and neural organization in autistic spectrum disorder. Frontiers in Human Neuroscience.

Nazari, M. A., Mosanezhad, E., Hashemi, T., \& Jahan, A. (2012). The effectiveness of neurofeedback training on EEG coherence and neuropsychological functions in children with reading disability. Clinical EEG and Neuroscience, 43(4), 315-322. http://dx.doi.org/10.1177/1550059412451880

Walker, J. E., Norman, C. A., \& Weber, R. K. (2002). Impact of qEEG-Guided coherence training for patients with a mild closed head injury. Journal of Neurotherapy, 6(2), 3143. http://dx.doi.org/10.1300/J184v06n02_05

\section{The Internalization of Neurofeedback Training as Demonstrated by Repeated QEEGs}

Corey Feinberg, MA, and Elsa Baehr, PhD

Neuro-Quest, Ltd., Illinois, USA

In general, neurofeedback training can be described as the process of using high-tech computer equipment to measure the electrical patterns of the brain (brainwaves) and then provide instantaneous feedback about those patterns for the purpose of enabling an individual to gain some level of influence or cognitive control over them (Hammond, 2011). In essence it is a process of self-remediation of unwanted activity in the brain. Typically changes in brainwave patterns occur gradually over time and with frequent rehearsal the changes become more enduring.

According to this conceptualization of the neurofeedback process, the first objective would be to learn the new, more desirable pattern of brainwave responses through a process of trial and error by relying on the feedback (audio and/or visual) for guidance. Once the individual has learned the intended brainwave pattern, it then becomes a matter of repetition of that pattern so that it may be properly "imprinted" in the brain in order to have some lasting result. Somewhere between the learning phase and the rehearsal phase, it can hypothesized that there is an internalization of the intended brainwave pattern by the individual in training and that they could learn to produce this pattern, at will, even without the aid of the feedback. It would then be reasonable to assume that the repetition of this newly internalized pattern would have increasingly more and more influence over the resting brain that was not actively engaged in the neurofeedback training process at the time, thereby indicating a lasting effect of a reconditioned brain. 
The purpose of this presentation is to provide empirical evidence of the internalization and remediation process that occurs during the course of neurofeedback training and treatment. Using multiple case examples and creative applications of Quantitative Electroencephalography (QEEG) analysis, this process can be illustrated in stages as they occur, providing a vivid cross-section of the changing brain showing both the internalization of normalized EEG patterns and the global improvements that occur along the way as compared to the resting state baseline QEEG measures. The clinical data presented will serve to negate any possible influence of the placebo effect as well as other therapeutic implications for accelerating treatment regimens and increasing efficacy of therapeutic outcomes from neurofeedback training efforts. One objective of this standing presentation is to demonstrate to the audience a creative manner of using QEEG for diagnostic purposes that may not have been commonly thought of before.

\section{References}

Hammond, D. C. (2011). What is neurofeedback: An update. Journal of Neurotherapy, 15(4), 305-336. http://dx.doi.org/10.1080/10874208.2011.623090

\section{Offering Hope: The Efficacy of Neuroptimal Training in the Treatment of Anxiety and Depression}

Janet McCulloch, MD, and Linda Beckett, MD

Kingston Institute of Psychotherapy and Neurofeedback, Canada

Kingston Institute of Psychotherapy and Neurofeedback was established in 2010 with the goal of making EEG-neurofeedback accessible in the Kingston community. Kingston is a small city with a large military base and is home to many large penitentiaries. Not surprisingly, there is a strong need for mental health care directed toward treatment for depression, anxiety, substance dependence, and post-traumatic stress disorder (PTSD). Neuroptimal is the mainstay of treatment at this multi-system clinic, which treats over 100 patients daily. The clinic also uses qEEG, targeted EEG, Hemo-encephalography, BAUD and Alpha-Stim. The 10 neurofeedback systems are supervised by two physicians.

Our patient population includes civilian, first responders, active serving military, and veterans of the Canadian Forces. Diagnostic groups routinely treated at the clinic include sleep disorders, PTSD, mood and anxiety, ADHD, and neurocognitive dysfunction. These patients invariably describe symptoms of anxiety and depression. For this reason, the clinic uses the Beck Depression Inventory (BDI) and the Beck Anxiety Inventory (BAI) to determine the effectiveness of intervention over time.

Study Design: This is a retrospective case series, which reviewed self-report outcome measures (BDI, BAl) at 0,8 , and 16 weeks. Patients were included only if they had no medication changes during this time period. Results for more than 200 patients demonstrate clear efficacy in decreasing disability symptoms of anxiety and depression. Results are analyzed based on:

- Symptom severity

- Age

- Gender

- Primary diagnosis 
Data from this retrospective case series suggest that Neuroptimal neurofeedback can be used as a primary intervention to alleviate symptoms of depression and anxiety. Individuals who suffer from PTSD describe high levels of anxiety and struggle with feelings of depression. Neuroptimal training benefits our patients with PTSD by decreasing subjective symptoms of anxiety and depression within this group.

\title{
Effectiveness of Pulsed, Ultra Low Power Electric Current EEG Biofeedback in Treating Symptoms of Concussions in Former NFL Players
}

\author{
George Rozelle, $P h D^{1}$, David Dubin, $M D^{2}$, and Fred Willis, $B B A^{3}$ \\ ${ }^{1}$ MindSpa Mental Fitness Center, Florida, USA \\ ${ }^{2}$ The Dubin Clinic, California, USA \\ ${ }^{3}$ HPN Neurologic, Massachusetts, USA
}

Football related traumatic brain injury $(\mathrm{TBI})$ is one of the signature sports injuries. Even one concussion can cause a lifetime of problems. TBI can result in a myriad of symptoms, including hypersensitivity, headaches, cognitive dysfunction, depression, and many more. Currently there is no adequate treatment. Little more than rest is generally prescribed. Residual symptoms can leave a person appearing outwardly normal but still have devastating consequences on the person and his family. While it has not yet been conclusively proven, it may be that repeated concussions lead to CTE, chronic traumatic encephalopathy. There have been numerous reports of mild/moderate TBI responding very well to this treatment. This has certainly been the experience of three Investigators on this project.

This project seeks to determine whether pulsed ultra-low power electric current EEG biofeedback is an effective treatment for symptoms of TBI. It is hypothesized, based on informal clinical observations, that this form of EEG biofeedback can have a significant and beneficial impact on problems associated with TBI such as anxiety, depression, sleep disorders, headaches, cognitive dysfunction, emotional ability, and other symptoms of TBI. This project will track across-session subjective symptoms ratings over the course of 20 sessions. There will also be pre- and post-testing using brain maps (qEEG), the Rivermead Post Concussion Questionnaire, the King-Devick test for cognitive functioning, a Sway/Balance for equilibrium and the ImPact test for cognitive function (memory, etc.). There will be follow-up testing 3 and 6 months after treatment has ended.

This study could be an important step toward validating a safe, effective, non-pharmaceutical intervention for a growing population of athletes who have sustained concussions and have residual symptoms. These symptoms can last a lifetime and have catastrophic effects on the athlete and his family. In addition, TBI resulting from repeated sports concussions is likely to not be so different from other causes, with the exception of blast injuries, which have their own particular mechanism of injury and pathophysiology. Verifying an effective treatment for this disorder would be a huge benefit to the approximately 1.7 million people each year who sustain a significant TBI. The ramifications of the study go far beyond treating athletes and even those in the military who are injured. Attendees will gain an understanding of the unique dynamics of reaching, evaluating, and treating former NFL players. 


\section{POSTER PRESENTATIONS}

\section{Lateralized Readiness Potential (LRP) as a Premotor Preparation Abnormality Biomarker in Autism and ADHD}

Estate Sokhadze, $P h D^{1}$, Stephen Edelson, $P h D^{2}$, Guela Sokhadze, BS ${ }^{1}$, Lonnie Sears, $P h D^{1}$, Yao Wang, $M S^{1}$, and Manuel Casanova, $M D^{1}$

${ }^{1}$ University of Louisville, Kentucky, USA

${ }^{2}$ Autism Research Institute, California, USA

Background. Motor abnormalities in autism bear the characteristics of precedence and universality that characterize a core symptom. Underlying the motor skill deficits in autism is a dyspraxia that by definition is an inability to plan, organize, and execute movements in the absence of any known physical and/or neurological condition (Mostofsky et al., 2006). This deficit is more apparent in autism spectrum disorders (ASD) when compared to ADHD.

Objectives. The lateralized readiness potential (LRP) is an index of motor processes and it is assumed that this brain potential is generated by a source within the motor cortex (Leuthold, Sommer, \& Ulrich, 2004). LRP reflects the response-specific involvement of the left and right cortices of the brain and enables the determination of the point in time at which the activation of the motor cortex controlling one hand surpasses the activation of the motor cortex controlling the other side (Eimer, 1998). The LRP is assumed to be related to selective response activation. It captures the asymmetric portion of the late Bereitschaftspotential preceding hand movements and helps to determine exact point in time when sensory information starts affect motor processing and execution.

Method. Our goal was to compare differences in LRP during a modification of a cued Posner spatial attention task between group of children with ASD, ADHD, and neurotypical (NT) children ( $N=14 /$ group, mean 14.9 years). Each subject participated in a Posner spatial attention task with congruent $(75 \%)$ and incongruent $(25 \%)$ trials with 128 -channel EEG recording. We used stimulus-locked LRP method of waveform calculation and since S1-S2 interval was set on 1s computed mean LRP and integrated LRP values for two windows (early $600-800 \mathrm{~ms}$ post-S1; and late $800-1200 \mathrm{~ms}$ post-S1). In addition we calculated N100, P200, and P300 to S2 stimuli.

Results and Conclusions. In our sample of ASD, ADHD, and NT controls, differences were not significant at the early stage, but became significant at the late stage of the LRP yielding Time (early, late) $X$ Group interaction $(F=6.77, p=0.012)$. Post-hoc analysis showed group differences at 800-1200 ms post-S1 window $(F=4.81, p=0.033)$ between ASD and NT groups. Autism group also showed more pronounced differences of LRPs in incongruent trials. Frontal N100 and P200 components showed delayed latencies in both ASD and ADHD as compared to controls $(F=4.72, p=0.03)$. Our study suggests more deficient motor preparation during task congruency manipulations in ASD as compared to NT and even ADHD, which was manifested in late LRP reflective of abnormal motor preparation processing, and delayed middle latency ERP components in response to incongruent targets. Premotor potentials such as LRP can be considered as useful biomarkers of motor coordination deficits and dyspraxia in autism. 


\section{References}

Mostofsky, S. H., Dubey, P., Jerath, V. K., Jansiewicz, E. M., Goldberg, M. C., \& Denckla, M. B. (2006). Developmental dyspraxia is not limited to imitation in children with autism spectrum disorders. Journal of the International Neuropsychological Society, 12(3), 314-326. http://dx.doi.org/10.1017/S1355617706060437

Eimer, M. (1998). The lateralized readiness potential as an on-line measure of central response activation processes. Behavior Research Methods, Instruments, \& Computers, 30(1), 146-156. http://dx.doi.org/10.3758/BF03209424

Leuthold, H., Sommer, W., \& Ulrich, R. (2004). Preparing for action: inferences from CNV and LRP. Journal of Psychophysiology, 18(2-3) 77-88.

http://dx.doi.org/10.1027/0269-8803.18.23.77

\section{Neuromodulation Based on 18 Session rTMS Course Improves Functional Measures of Information Processing and Behavioral Responses in Autism Spectrum Disorder}

Estate Sokhadze, PhD, Ayman El-Baz, PhD, Lonnie Sears, PhD, and Manuel Casanova, MD University of Louisville, Kentucky, USA

Background. The study is based on an underlying neuropathology model of autism, which emphasizes minicolumnar pathology and lateral inhibition deficits resulting in behavioral and executive dysfunctions. We proposed that neuromodulation based on low frequency repetitive Transcranial Magnetic Stimulation (rTMS) will enhance lateral inhibition through activation of inhibitory double bouquet interneurons and will be accompanied by improvements in the prefrontal executive functions. In addition, we proposed that rTMS will improve cortical excitation/inhibition ratio and result in changes manifested in electrocortical responses such as event-related potentials (ERP) and evoked and induced EEG gamma oscillations during cognitive tests.

Objectives. TMS offers a noninvasive method for altering excitability of the neural circuits and induction of functional reorganization in the cortex. We reported earlier positive effects of rTMS in autism spectrum disorders (ASD) in our pilot studies using shorter 6- and 12session long rTMS courses (Sokhadze et al., 2009, 2010, 2012; Casanova et al., 2012; Baruth et al., 2010). Along with traditional behavioral evaluations in current study we used ERPs and induced gamma responses power and coherence in a visual oddball task with illusory Kanizsa figures. We compared clinical, behavioral, and electrocortical (ERP, single trial EEG) outcomes in two groups of children with autism (TMS, wait-list group). We predicted that 18-session long course in ASD patients will have better behavioral and ERP/EEG outcomes as compared to an age- and IQ-matched wait-list ASD group.

Methods. We used 18 sessions of $1 \mathrm{~Hz}$ rTMS applied bilaterally over the dorso-lateral prefrontal cortex (DLPFC) in 27 individuals with ASD (10-21 years old). The wait-list group was comprised of 20 age-matched subjects with ASD tested twice. Both the TMS and waitlist (WTL) groups were assessed at (1) the initial baseline using clinical behavioral questionnaires (i.e., Aberrant Behavior Checklist $[A B C]$, and Repetitive Behavior ScaleRevised [RBS-R]) and during performance on visual oddball task with Kanizsa illusory figures, and (2) post-completion of 18 sessions of TMS (or wait period). 
Results. Post-TMS evaluations showed decreased irritability and hyperactivity on the $A B C$, and decreased stereotypic behaviors on the RBS-R. Following rTMS course we found decreased magnitude of the frontal N100 ERP component and evoked gamma power to nontargets, and shorter of the fronto-central P2a and P3a ERPs and reduced induced gamma oscillation power to non-targets in TMS group as compared to the WTL. These ERP changes along with increased $\mathrm{P} 3 \mathrm{~b}$ and enhanced induced gamma oscillations (higher power, phase coherence) to targets are indicative of more efficient processing of information posttreatment. Enhanced information processing was also reflected in such behavioral response measures as reaction time (RT) and error rate.

Conclusion. These results could be considered as a replication of our prior studies using shorter rTMS course. Collected data support our concept that rTMS improves executive functioning as evidenced by normalization of ERP and EEG responses and behavioral reactions during executive function test, and also by improvements in clinical behavioral evaluations. The study links behavioral, clinical, and ERP/EEG responses during cognitive tests and TMS outcomes with an underlying developmental neuropathology model derived from investigations in our laboratory.

\section{References}

Baruth, J. M., Casanova, M. F., El-Baz, A., Horrell, T., Mathai, G., Sears, L., \& Sokhadze, E. (2010). Low-frequency repetitive transcranial magnetic stimulation modulates evoked-gamma frequency oscillations in autism spectrum disorders. Journal of Neurotherapy, 14(3), 179-194.http://dx.doi.org/10.1080/10874208.2010.501500

Casanova, M. F., Baruth, J. M., El-Baz, A., Tasman, A., Sears, L., \& Sokhadze, E. (2012). Repetitive transcranial magnetic stimulation (rTMS) modulates event-related potential (ERP) indices of attention in autism. Translational Neuroscience, 3(2), 170-180. http://dx.doi.org/10.2478/s13380-012-0022-0

Sokhadze, E. M., El-Baz, A., Baruth, J., Mathai, G., Sears, L., \& Casanova M. F. (2009). Effects of low-frequency repetitive transcranial magnetic stimulation (rTMS) on induced gamma frequency oscillations and event- related potentials during processing of illusory figures in autism. Journal of Autism and Developmental Disorders, 39(4), 619-634.

Sokhadze, E., Baruth, J., Tasman, A., Mansoor, M., Ramaswamy, R., Sears, L., ... Casanova, M. F. (2010). Low-frequency repetitive transcranial magnetic stimulation (rTMS) affects event-related potential measures of novelty processing in autism. Applied Psychophysiology and Biofeedback, 35(2), 147-161. http://dx.doi.org/10.1007/s10484-009-9121-2

Sokhadze, E. M., Baruth, J. M., Sears, L., Sokhadze, G. E., El-Baz, A. S., \& Casanova, M. F. (2012). Prefrontal neuromodulation using rTMS improves error monitoring and correction functions in autism. Applied Psychophysiology and Biofeedback, 37(2), 91-102. http://dx.doi.org/10.1007/s10484-012-9182-5 


\title{
EEG Biofeedback Benefits Acquisition and Consolidation of Motor Sequence
}

\author{
Roman Rozengurt, $M A^{1}$, Sunao Uchida, Prof. ${ }^{2}$, Anat Barnea, $D S c^{3}$, and Daniel Levi, Senior \\ Lecturer $^{1}$ \\ ${ }^{1}$ Department of Psychology, Interdisciplinary Center Herzliya, Israel \\ ${ }^{2}$ Faculty of Sport Science, Waseda University, Japan \\ ${ }^{3}$ Neurofeedback practitioner, Biokeshev, Israel
}

The evidence from different studies showed the importance of night sleep in consolidation of procedural memory. Our study tries to find out whether consolidation processes can be achieved by neurofeedback training.

It is hypothesized that consolidation of motor learning during sleep is associated with theta band activity. We used EEG-biofeedback (NF) to train participants (30 subjects, divided into two groups) to selectively increase either their relative theta or beta band power following the acquisition phase of motor sequence learning. We employed the keyboard version of the finger-tapping task (FTT) for motor learning. The participants were instructed to type the predefined order of letters as quick and accurate as possible. We tested performance on a motor task before and after motor training, after one NF session, one day, and one week.

By testing the performance in each those phases we could explore the interaction between immediate NF and subsequent sleep-dependent consolidation processes. Results revealed a significant improvement in performance immediately after NF in the theta group, but not in the beta group. The rate of improvement was significantly higher at theta group (more than $10 \%)$ in compare to beta group $(4 \%)$.

Although participants were provided with feedback regarding the change in target band power using a single electrode (Fz for the beta group; Pz or Fz for the theta group, which did not differ in their modulation of theta power), in the current study we were able to measure the extent to which that single-channel feedback caused more widespread changes in the other 18 electrodes from which EEG was recorded.

The theta effect was kept up to at least one week following training: the performance slightly improved on the tests of second day and seventh day in the theta group and slightly decreased in the beta group. Across participants, post-NF improvement positively correlated with theta/beta ratio achieved during NF training in the theta group, but not in the beta group, indicating a clear relationship between memory consolidation, as reflected in motor performance, and theta NFT. Additional analysis showed correlation between EEG changes at target electrode with changes at other electrodes. Thus, regulating theta power may yield contributions to the initial performance and subsequent consolidation of an acquired motor skill.

\section{A Pilot Study on the Correlates of Sleep Problems in Chronic Pain Patients}

Tina Tin, MSc, and Gabriel Tan, PhD

National University of Singapore, Singapore

Although traditional medical treatment has been relatively successful for treating acute pain, its record for treating chronic pain has been quite limited in efficacy and the findings inconsistent and inconclusive. Alternative treatments have been introduced and empirically 
validated, many with promising results. Among these are non-pharmacological neuromodulatory (NPNM) approaches such as brain stimulation, hypnosis, biofeedback, and neurofeedback (Tan et al., 2010). A number of these modalities have received empirical support in the Western literature. However, its generalizability and applicability to individuals with chronic pain outside of the United States is not known. A pilot study to assess the feasibility, acceptability, and relative efficacy of four NPNM modalities (self-hypnosis, cranial electrical simulation, HRV biofeedback, and cognitive behavioral therapy) for pain management was initiated in Singapore in 2012 and is still ongoing.

The data for this poster was obtained via the above described research project. This paper explored psychosocial correlates of chronic pain in the Singapore population, with a particular focus on sleep problems, pain catastrophizing, and negative and positive affect. Building on the findings of Buenaver et al. (2006), three mediation models were tested using a bootstrapping technique. Model 1 was a replication and examined the indirect effects of pain catastrophizing through sleep problems on pain outcomes (intensity and interference). Model 2 extended the previous research and assessed the indirect effect of negative and positive affect (conceptualized as a ratio) through sleep problems on pain outcomes. Model 3 linked the three variables and examined the indirect effect of pain catastrophizing through affect on sleep problems. For the study, 33 patients with chronic pain in the lower back or knees were recruited from the National University Hospital, a tertiary teaching hospital in Singapore.

Pain interference was shown to be more strongly associated with other variables than pain intensity. Pain catastrophizing, sleep problems, and negative affect had significant correlations with other variables, while positive affect generally showed no significant associations. Model 1 was only supported for the indirect effects of pain catastrophizing (magnification) on pain interference. Model 3 was supported for pain catastrophizing and all its three components of rumination, magnification, and helplessness. The findings show the importance of considering psychosocial factors for chronic pain patients in Singapore, and that pain catastrophizing and negative affect should be addressed in the treatment of sleep problems for chronic pain patients.

\section{References}

Tan, G., Jensen, M. J., Dao, T., Stoelb, B., \& Gunkelman, J. (2010). Nonpharmacological neuromodulatory approaches to pain management. In R. Kerns \& M. Ebert (Eds.), Behavioral and Psychopharmacological Therapeutics in Pain Management, Cambridge University Press.

\section{Looking for Alternative Screening Measures for Use on National Depression Screening Day}

Tom Golightly, PhD, Jon Cox, PhD, Dallas Jensen, PhD, Tanner Carlock, and Andrea McCall Brigham Young University Counseling and Psychological Services, Utah, USA

National Depression Screening Day (NDSD) has been held since 1991 with approximately 500,000 individuals screened yearly. Many screening sites located at college counseling centers typically order a combination of screening tools. These four measures usually include: the Hands Depression Screening Tool (Bear et al., 2000); the Carroll-Davidson generalized anxiety screener (Carroll \& Davidson, 2000); the Mood Questionnaire for bipolar disorder (Seetal et al., 2009); and the SPRINT-4 post-traumatic stress disorder screen (Soberay, Faragher, Barbash, Brookover, \& Grimsley, 2013). The Outcome Questionnaire- 
45.2 (OQ-45; Lambert et al., 2004) is considered the gold standard of outcome measures and has been widely studied, demonstrating solid reliability and validity.

In the current study of 285 students, the OQ-45 was used as a screening tool in addition to the NDSD tools. Preliminary results indicate that the Hands Depression Screening Tool correlates significantly $(r=.81)$ with the OQ-45 total scores. The Carroll-Davidson generalized anxiety screener also correlates significantly $(r=.74)$ with the OQ-45 total scores.

It is clear that the OQ-45 might be effectively used as a screening tool in college populations. NDSD screeners are also used at a cost to most counseling centers, whereas administering additional OQ-45's for screening purposes might save some operating cost and do as well at screening individuals wondering about their mental health.

Since the mid 1990s BYU CAPS has utilized a 45-item measure of psychological distress with clients, the Outcome Questionnaire-45 (OQ-45). The OQ-45 also takes about 7 to 10 minutes to complete. It is considered the gold standard of outcome measures and has been widely studied, demonstrating solid reliability and validity. The screening measures used in NDSD are not as widely studied. CAPS faculty and staff must train and re-train to use the Hands Screening tool each year. There is also financial cost associated with using the traditional NDSD screening tools. Anecdotally, CAPS clinicians have noticed that the screening tools may be too sensitive (a threat to validity), creating too many false positives for mental health conditions. Based on the results of the screenings, a high number of referrals to BYU CAPS are made which creates a backlog of students trying to be seen by CAPS clinicians immediately following NDSD. The current investigation looks to see if the $\mathrm{OQ}-45$ could be used as a more reliable and valid screening tool. If the OQ-45 could be used as an NDSD screener, we could perhaps be more effective in giving at-risk students referrals to counseling, save money and time of CAPS clinicians, and help alleviate some of the clinical demand placed on CAPS immediately following NDSD.

The questions asked included: Do individuals who fall in the clinically significant range of the NDSD screening measures also fall in the clinical range on the OQ-45? If not, what are the differences? Are there any patterns to false positives (or false negatives)? Will the OQ-45 be an accurate and efficient screening measure to use during NDSD events?

\section{References}

Bear, L., Jacobs, D. G., Meszler-Reizes, J., Blais, M., Fava, M., Kessler, R., ... O'Laughlen, J. (2000). Development of a brief screening tool: the HANDS. Psychotherapy and Psychosomatics, 69(1), 35-41.

Carroll, B. J., \& Davidson, J. R. T. (2000). Screening Scale for DSM-IV, Generalized Anxiety Disorder.

Lambert, M. J., Morton, J. J, Hatfield, D., Harmon, C., Hamilton, S., Reid, R. C., ... Burlingame, G. B. (2004). Administration and Scoring Manual for the Outcome Questionnaire-45. Orem, UT: American Professional Credentialing Services. 
Seetal, D., Williams, L. J., Jacka, F. N., Pasco, J. A., Bjerkeset, O., \& Berk, M. (2009). Reliability of the Mood Disorder Questionnaire: Comparison with the Structured Clinical Interview for the DSM-IV-TR in a population sample. Australian and New Zealand Journal of Psychiatry, 43(6), 526-530.

Soberay, A., Faragher J. M., Barbash, M., Brookover, A., \& Grimsley, P. (2013). Pathological Gambling, Co-occurring Disorders, Clinical Presentation, and Treatment Outcomes at a University-Based Counseling Clinic. Journal of Gambling Studies, 30(1), 61-69. http://dx.doi.org/10.1007/s10899-012-9357-2

\section{Effects of Neurofeedback Training in a Group of Adolescents in Conflict with the Law in Sonora Mexico}

Teodoro Hernandez, MS, and Maria Eugenia Gãemez, MD

A Mas Centros, Mexico

Background and Description. Juvenile delinquency is a problem that affects any society. According with the U.S. Department of Justice, 1,470,000 juvenile arrests were registered in this country in 2011 (Puzzanchera, 2013), and in Mexico, the state of Sonora has the most adolescent population in internment (INEGI, 2012). According to some studies, antisocial and criminal behavior is associated with attention deficit, learning problems, and impulsivity (Barkley, 1997). One method that has been utilized is neurofeedback (NFB) training (Monastra et al., 2006); however, at the present time there is not much information about the effects of this procedure in adolescents in conflict with the law, nor information about the impact of the NFB in this population in Mexico.

Objective. The objective of this study is to determine changes in performance on the WISCIV and the Stroop task (Golden, 2001), in a group of adolescents in conflict with the law, after the implementation of NFB protocols.

Methods. Participants consisted of 9 male adolescents. The group ranged in ages from 14 to 16 years. All participants were inmates of an internment center at the city of Hermosillo Sonora, Mexico. The training protocol selected consisted of the increase of beta waves, alpha waves and sensorimotor rhythm (SMR), in 48 sessions of 20 minutes each. In the sessions that increased alpha waves, the participants were relaxed and with their eyes closed. The WISC-IV and the Stroop task (Golden, 2001) were applied before and after the training protocol.

Results. Pre- to post-training scores were compared. Paired sample $t$-tests were performed to assess changes in pre- and post-training scores from the WISC-IV and Stroop task.

Significant differences were found in the four indeces of the WISC-IV: Verbal Comprehension $(p<.001)$; Perceptual reasoning $(p<.001)$; working memory $(p<.01)$ and processing time $(p$ $<.01)$ as well as in the $\mathrm{Cl}$ index $(p<.001)$. On the other hand, in the Stroop task significant differences were found in reading the words, naming the color, and naming the color instead of reading the words.

Conclusion. This is a preliminary study about the effects of NFB training in adolescents in conflict with the law in Mexico; however, more investigation and more subjects are necessary to generalize these results with this kind of population and to collaborate with the social reinsertion of these adolescents. 


\section{References}

Barkley, R.A. (1997). Behavioral inhibition, sustained attention, and executive functions: Constructing a unifying theory of ADHD. Psychological Bulletin, 121(1), 65-94.

Golden, C.J. (2001). Stroop: Test de colores y palabras. (3rd Ed.). Madrid: TEA Ediciones.

Instituto Nacional de Estadistica Geografica e Informatica. (INEGI). (2012). Menores Infractores. Porcentaje de menores infractores en diagnostico, en tratamiento interno y en tratamiento externo por entidad federativa según sexo. Recuperado el 4 de julio del 2012.

http://www.inegi.org.mx/prod_serv/contenidos/espanol/bvinegi/productos/integracion/ pais/aepef/2013/aeg pef_2013

Monastra, V. J., Lynn, S., Linden, M., Lubar, J. F., Gruzelier, J., \& La Vaque, T. J. (2006). Electroencephalographic biofeedback in the treatment of attentiondeficit/hyperactivity disorder. Journal of Neurotherapy, 9(4), 5-34. http://dx.doi.org/10.1300/J184v09n04_02

Puzzanchera, C. (2013). Juvenile Arrests 2011. Juvenile Offenders and Victims National Report Series. Washington DC: Office of Juvenile Justice and Delinquency Prevention.

\section{Examining the Cognitive Benefits of a Phonemic Intelligence Program in Older Adults with Mild Cognitive Impairment}

Rebecca Prostko, $M D^{1}$, Bernadette Lange, $P h D^{2}$, and Baskaran Pillai, $P h D^{3}$

${ }^{1}$ Private Practice, Florida, USA

${ }^{2}$ Florida Atlantic University, Florida, USA

${ }^{3}$ Pillai Center for Mind Science, Pennsylvania, USA

Background and Description. The incidence and prevalence of Mild Cognitive Impairment (MCl) is difficult to estimate (Ward, Arrighi, Michels, \& Cedarbaum, 2012). Yet many older adults express concerns to practitioners during office visits about memory issues. Current treatment of $\mathrm{MCl}$ focuses on diet, exercise, lifestyle changes, as well as social and intellectual stimulation (Cooper, Li, Lyketsos, \& Livingston, 2013). Web-based memory exercise programs are available (Fortman, 2012) although older adults may lack Internet access and computer skills. The office visit is the opportunity for practitioners to engage older adults to discuss in-office interventions such as the complementary and alternative modalities (CAM) of neurofeedback and in-home interventions of Phonemic Intelligence exercise (PIE). PIE is a process of producing human sounds while positioning the hands on the head in a specific pattern to increase energy to targeted areas of the brain (Pillai). The purpose of this study is to explore the effectiveness of PIE for older adults who are experiencing alterations in memory.

Methods. Older adults who self-identify as experiencing memory changes are volunteers for the study. Exclusion criteria included persons taking seizure or anti-psychotic medications, a history of seizures, or active major expression. A quasi-experiential design is used with an anticipated sample of intervention $n=50$ and control $n=50$. A rolling enrollment during a 6 month period is being used. Subjects volunteer for an 8-week period in study. All subjects 
complete the Geriatric Depression Scale, Memory Assessment Scales, and QEEG at the beginning and end of the 8-week period. The intervention group is taught PIE and instructed to practice PIE twice daily and record in a log.

Results. Data collection and active recruitment is in progress. $N=45$ total participants with a low attrition rate.

Conclusion. QEEGs will be analyzed via z-scores and ANOVA pre- and post-intervention using NeuroGuide software. Areas of neuronal de-regulation will be reevaluated at the end of the study to assess whether the Phonemic Intelligence program improved functioning in the de-regulated areas of the brain. Pre- and post-MAS will be scored.

\section{References}

Cooper, C., Li, R., Lyketsos, C., \& Livingston, G. (2013). Treatment for mild cognitive impairments: Systematic review. British Journal of Psychiatry, 203(3), 255-264. http://dx.doi.org/10.1192/bjp.bp.113.127811

Fortman, J. (2012). Computer-Based Cognitive Training for Age-Related Cognitive Decline and Mild Cognitive Impairment. (Electronic Thesis or Dissertation). Retrieved from https://etd.ohiolink.edu/

Ward, A., Arrighi, H. M., Michels, S., \& Cedarbaum, J. M. (2012). Mild cognitive impairment: Disparity of incidence and prevalence estimates. Alzheimer's \& Dementia: The Journal of the Alzheimer's Association, 8(10), 14-21. http://dx.doi.org/10.1016/j.jalz.2011.01.002.

\section{Discrimination of EEG Gamma (36-50 Hz) Activity in Wernicke's Area While Listening to an Audio Book}

Kyle Weiner, Audra Turner, Sabrina Tom, and Jon Frederick, PhD

Lindsey Wilson College, Kentucky, USA

Previous studies have found that about three fourths of human subjects can discriminate EEG alpha activity (Frederick, 2012; Kamiya, 1968), but relatively few can do it consistently across 10 sessions. Since gamma activity has previously been shown to be involved in conscious awareness of sensory information (Engle \& Singer, 2001), we hypothesized subjects could reliably discriminate gamma $(36-50 \mathrm{~Hz})$ activity in Wernicke's area (Brodmann Area 22; left hemisphere). We also hypothesized that listening to an audio book during the discrimination task might be a more naturalistically valid condition for attending to activity in this region, and result in better performance than a quiet eyes-closed condition. Eight participants completed a total of 35 (median 4) sessions. Each second, a 90-second baseline was recorded. Eyes were closed during the baseline and task conditions. During the task, low (< 40th percentile of baseline) and high gamma events ( $>60$ th percentile) triggered a prompt that participants then responded to using a high or low key press, and received immediate feedback on their answer. For the first half of odd-numbered sessions, participants listened to an audio book. Two participants achieved criterion performance (binomial $p<.01$ ). Consistent with psychophysics, these participants were better able to discriminate very high and low signal intensities as compared to moderately high and low 
intensities. In the four odd-numbered sessions during which participants reached criterion, performance improved in the second half of the session after the audio book had been turned off. This contradicted our hypothesis and suggested that the audio book might have served as more of a distraction than a relevant stimulus. However, a comparison to the even numbered sessions did not support this interpretation. In conclusion, only $25 \%$ of subjects able to discriminate gamma power in Wernicke's area within five sessions in this study. Future studies will assess whether providing standard neurofeedback training during half of each session may improve EEG state discrimination performance (as suggested by Cinciripini, 1984; Kotchoubey, Kubler, Strehl, Flor, \& Birbaumer, 2002).

\section{References}

Cinciripini, P. M. (1984). Discrimination of sensorimotor EEG (12-25 Hz) activity: A comparison of response, production, and no-feedback training conditions. Psychophysiology, 21(1), 54-62.

Engle, A. K., \& Singer, W. (2001). Temporal binding and the neural correlates of sensory awareness. Trends in Cognitive Sciences, 5(1), 16-25. http://dx.doi.org/10.1016/S1364-6613(00)01568-0

Frederick, J. A. (2012). Psychophysics of EEG alpha state discrimination. Consciousness and Cognition, 21(3), 1345-1354. http://dx.doi.org/10.1016/j.concog.2012.06.009

Kamiya, J. (1968). Conscious control of brain waves. Psychology Today, 1, 57-60.

Kotchoubey, B., Kubler, A., Strehl, U., Flor, H., \& Birbaumer, N. (2002). Can humans perceive their brain states? Consciousness and Cognition, 11(1), 98-113. http://dx.doi.org/10.1006/ccog.2001.0535

\section{One Successful Case of Neurofeedback Sessions for Social Phobia and Academic Performance}

Gabriel Tan, $P h D^{1}$, Jayun Choi, $B A^{2}$, and Takashi Obana, $M A^{1}$

${ }^{1}$ National University of Singapore, Singapore

${ }^{2}$ Han Kook Chon, Singapore

Feelings of nervousness and anxiety in social settings always vexed Client A. Her past psychological trauma, which was inflicted upon her over an extended period of time, made her extremely difficult to feel at ease when she had to deal with interpersonal issues. Client A had been attempting various methods to overcome this difficulty, including counseling sessions or a medical leave. However, nothing seemed to have substantial efficacy on improving her perceived symptoms. In dire need to survive within a very competitive academic setting, she sought a therapy using neurofeedback technique. The therapy involved T3-T4 Delta, Pz Alpha, P4 Beta, and C3 Beta. After the first month of T3-T4 Delta (intended for a brain recovery) and P4 Beta (intended to improve reading other people's emotions) trainings, she discovered herself interacting freely with whomever came across her life. In the second month of therapy, C3 Beta training (intended to eliminate persistent daydreaming) was added. During this period, she felt that she was able to focus on the difficult lectures with such ease. Although her study hours decreased due to crammed schedule hunting for a job, the grades obtained in exams and assignment improved 
compared to the previous semesters. In the third month, Pz Alpha training was added to boost her peak performance because job interviews were awaiting her. To her surprise, she was able to present herself and her competency without being self-conscious, let alone being neither nervous nor anxious. The job hunting went extremely successfully and she now has a great work-life balance with a strong sense of subjective wellbeing. Several implications could be delineated from this case study. The client showed some resistance toward traditional counseling sessions. The fact that counseling sessions involve prolonged vis-à-vis contact bore too much burden for Client A. Every time the counseling sessions were attempted, they were terminated prematurely. The alternative method of mental training, such as Vipassana meditation, was even detrimental to her wellbeing since it induced nightmares and other dreadful emotions. Taken together, neurofeedback was suitable for this case because 1) it does not involve prolonged vis-à-vis contact, and 2) it can be altered for mental training techniques (such as meditation) without tapping into the deep psyche. 